\title{
Regularity of solutions for some problems of mathematical physics
}

\author{
by
}

\author{
A. KOSHELEV \\ Univ. Stuttgart Mathematisches Institut A \\ 801140 Stuttgart, D-70511 Germany \\ and \\ St. Petersburg Univ. Fac. of Math. \\ and Mech. 1 Ulianovskayast. \\ 198904 St. Petersburg-Petrodvoretz Russia.
}

ABSTRACT. - Coercivity estimates of solutions for some problems of mathematical physics including parabolic and Stokes systems are obtained.

Key words: Elliptic parabolic systems.

\section{PREFACE}

This paper is devoted to some boundary value problems for systems of partial differential equations. In particular we consider Stokes system and quasilinear elliptic degenerate systems of divergent type with bounded nonlinearities. The author would like to express his deep gratitude to Prof. S. Hildebrandt for fruitfull discussions and general support.

It was shown in [3] (see also [4] and [9]) that the question of regularity of weak solutions for quasilinear elliptic and parabolic systems is closely attached to the dispersion of the spectrum of the matrix which defines the ellipticity (parabolicity) of the system. The upper bound for this dispersion is determined by some coercive constants for elementary elliptic or parabolic operators. The explicit form of these constants leads to some conditions which are easy to check in order to obtain the regularity of weak solutions. This approach can be applied for example to such important systems as the Stokes system. We divide the paper in three sections. 
The first one is devoted to some constants concerning the operators $\Delta$ and $\varepsilon \partial_{t}-\Delta$, where $\varepsilon$ is an arbitrary positive constant.

Let $B$ be a unit ball in $R^{m}(m \geq 2)$ with the center at the origin and let $\alpha=2-m-2 \gamma(0<\gamma<1)$. If $u(x)$ is equal zero on $\partial B$ then the inequality

$$
\begin{aligned}
\int_{B}\left|D^{2} u\right|^{2}|x|^{\alpha} d x \leq & {\left[1+\frac{m-2}{m+1}+0(\gamma)\right] \int_{B}|\Delta u|^{2}|x|^{\alpha} d x } \\
& +C\left(\int_{B}\left|D^{2} u\right|^{2}|x|^{\alpha} d x\right)^{\frac{m}{m+2 \gamma}}\left(\int_{B}|D u|^{2} d x\right)^{\frac{2 \gamma}{m+2 \gamma}}
\end{aligned}
$$

holds true. Here $\left|D^{2} u\right|^{2}$ and $|D u|^{2}$ are, respectively, the sums of all squared derivatives of $u$ of the second and the first order. An analogous result was at first obtained by H. O. Cordes [1].

This estimate could be also obtained with the help of the result of E. Stein [4] concerning the boundness of the singular integral operators in the weighted spaces $L_{2, \alpha}\left(R^{m}\right)(|\alpha|<m)$. But this method doesn't give the explicit constant in front of the right-hand side integral containing $\Delta u$.

The nonstationary case is also considered in this section. Let $Q=$ $(0, T) \times B, u=0$ for $t=0$, and $\zeta$ is a cut-off function. Then the inequality

$$
\begin{aligned}
& \int_{Q}\left|D^{2} u\right|^{2}|x|^{\alpha} \zeta d x d t \\
& \leq \frac{m}{2}\left[1+\frac{m-2}{m+1}+0(\gamma)\right] \int_{Q}\left|\varepsilon \partial_{t} u-\Delta u\right|^{2}|x|^{\alpha} \zeta d x d t \\
&+C\left\{\left(\int_{Q}\left|D^{2} u\right|^{2}|x|^{\alpha} \zeta d x d t\right)^{\frac{m}{m+2 \gamma}}\left[\int_{Q}\left(|D u|^{2}+|u|^{2}\right) d x d t\right]^{\frac{2 \gamma}{m+2 \gamma}}\right. \\
&\left.+\int_{Q}\left(|D u|^{2}+|u|^{2}\right) d x d t\right\}
\end{aligned}
$$

holds for $m \geq 3$, and the constant $C$ doesn't depend on $\varepsilon>0$.

Section 2 is devoted to some coercivity estimates. In section 3 we consider the Stokes system both for stationary and nonstationary cases. Consider for example here only the stationary system

$$
\begin{cases}\Delta u+\nabla p & =f \\ \operatorname{div} u & =0\end{cases}
$$

in a bounded domain $\Omega \subset R^{m}$ with a smooth boundary and with $u=0$ on $\partial \Omega$.

Let $x_{0}$ be an arbitrary point of $\Omega$, with dist $\left(x_{0}, \partial \Omega\right)>R_{0}=$ const and $R<R_{0}$. 
Then the estimates for the weak solution $u, p$

$$
\begin{aligned}
& \int_{B_{R}\left(x_{0}\right)}|\nabla p|^{2}\left|x-x_{0}\right|^{\alpha} d x \\
& \quad \leq\left[1+\frac{(m-2)^{2}}{m-1}+0(\gamma)\right] \int_{B_{R}\left(x_{0}\right)}|f|^{2}\left|x-x_{0}\right|^{\alpha} d x+C \int_{B_{R}\left(x_{0}\right)}|f|^{2} d x
\end{aligned}
$$

and

$$
\begin{aligned}
& \int_{B_{R}\left(x_{0}\right)}\left|D^{2} u\right|^{2}\left|x-x_{0}\right|^{\alpha} \zeta d x \\
& \leq \quad\left\{1+\left[1+\frac{(m-2)^{2}}{m-1}\right]^{1 / 2}\right\}^{2}\left[1+\frac{m-2}{m+1}+0(\gamma)\right] \\
& \quad \times \int_{B_{R}\left(x_{0}\right)}|f|^{2}\left|x-x_{0}\right|^{\alpha} \zeta d x \\
& +C\left[\left(\int_{\Omega}\left|D^{2} u\right|^{2}\left|x-x_{0}\right|^{\alpha} \zeta d x\right)^{\frac{m}{m+2 \gamma}}\left(\int_{\Omega}|D u|^{2} d x\right)^{\frac{2 \gamma}{m+2 \gamma}}\right. \\
& \left.\quad+\int_{\Omega}\left(|D u|^{2}+|u|^{2}+|f|^{2}\right) d x\right]
\end{aligned}
$$

hold true and $C$ doesn't depend on $x_{0}$. The results of this paragraph were obtained in cooperation with A. Wagner (Cologne).

The third paragraph contains some results about the elliptic system

$$
\sum_{i=1}^{m} D_{i} a_{i}(x ; u, D u)-a_{0}(x ; u, D u)=0 .
$$

Under natural analytic conditions on the coefficients $a_{i}(x, p)$ we assume that the eigenvalues $\lambda_{j}$ of the symmetric matrix

$$
A=\left\{\frac{\partial a_{i}}{\partial p_{j}}\right\} \quad(i, j=0, \ldots, m)
$$

satisfy the following inequalities

$$
\frac{\lambda}{1+|p|^{s}} \leq \lambda_{j} \leq \frac{\Lambda}{1+|p|^{s}}
$$

with $\Lambda, \lambda=$ const. $>0$ and $0 \leq s<1$.

It is proved, for example, that if the inequality

$$
\frac{\left(1+\frac{m-2}{m+1}\right)[1+(m-2)(m-1)]}{\left(1+\frac{m-2}{m+1}\right)[1+(m-2)(m-1)]-1} \frac{\lambda}{\Lambda}>1
$$

holds then the "small" weak solution of the system satisfies the Hölder condition in $\bar{\Omega}$. 


\section{SOME COERCIVITY INEQUALITIES WITH EXPLICIT CONSTANTS}

Consider in $R^{m} \quad(m \geq 2)$ a ball $B_{R}\left(x_{0}\right)$ with the center $x_{0}$ and radius $R$. The ball $B_{1}(0)$ will be denoted $B$. In this ball an equation

$$
\Delta u=f(x)
$$

with a boundary condition

$$
\left.u\right|_{\partial B}=0
$$

is given.

Suppose that $f \in L_{2, \alpha}(B)$, where $L_{2, \alpha}$ is the space of squared integrable functions with a weight $|x|^{\alpha}$. Throughout this paper we assume that $\alpha=2-m-2 \gamma(0<\gamma<1)$, and $|x|$ denotes the distance from the origin. The norm in $L_{2, \alpha}(B)$ as usual is determined by

$$
\left(\int_{B}|u|^{2}|x|^{\alpha} d x\right)^{\frac{1}{2}}
$$

Set

$$
|D u|^{2}=\sum_{i=1}^{m} u_{i}^{2} \quad \text { and } \quad\left|D^{2} u\right|^{2}=\sum_{i, k=1}^{m} u_{i k}^{2},
$$

where $u_{i}$ are the derivatives with respect to $x_{i}$.

By $W_{2, \alpha}^{(2)}(B)$ we shall denote those functions in the Sobolev space $W_{2}^{(2)}(B)$ whose second derivatives are square summable with the weight $|x|^{\alpha}$. As norm in this space we could take for example the expression

$$
\left(\int_{B}\left|D^{2} u\right|^{2}|x|^{\alpha} d x+\int|u|^{2} d x\right)^{\frac{1}{2}} .
$$

One of the aims of this section is to prove for the solution of the problem (2.1), (2.2) the inequality

$$
\int_{B}\left|D^{2} u\right|^{2} r^{\alpha} d x \leq C_{\alpha}^{2} \int_{B}|f|^{2} r^{\alpha} d x, \quad(r=|x|)
$$

where $C_{\alpha}$ has an explicit form. For $\alpha^{\prime}=m-2+2 \gamma$ such an inequality was proved by the author in [2].

First we shall prove some lemmas. 
Lemma 2.1. - If $u \in W_{2, \alpha}^{(2)}(B)$, then the inequalities

$$
|u(0)|^{2}<\eta \int_{B}|D u|^{2} r^{\alpha} d x+C_{0}(\eta) \int_{B}|u|^{2} d x
$$

and

$$
\sum_{i=1}^{m}\left|u_{i}(0)\right|^{2}<\eta \int_{B}\left|D^{2} u\right|^{2} r^{\alpha} d x+C_{0}(\eta) \int_{B}|u|^{2} d x
$$

hold.

Here $\eta$ is as usual an arbitrary positive constant and

$$
C_{0}(\eta)=2 m|S|^{-(m / 2 \gamma+1)} \gamma^{-\frac{m}{2 \gamma}} \eta^{-\frac{m}{2 \gamma}}
$$

where $|S|$ is the surface of the unit sphere in $R^{m}$ and $\lambda$ is the smallest absolute value of the eigenvalues for operator $\Delta$ in $B$ with the condition (2.2).

Proof. - Evidently

$$
u(0)=u(x)-\int_{0}^{r} \frac{\partial u}{\partial \varrho} d \varrho .
$$

Square both sides of this equality and integrate over the ball $B_{\delta}(0)=B_{\delta}$ with $\delta<1$. We get

$$
|u(0)|^{2}|S| m^{-1} \delta^{m} \leq 2 \int_{B_{\delta}}\left|\int_{0}^{r} \frac{\partial u}{\partial \varrho} d \varrho\right|^{2} d x+2 \int_{B} u^{2} d x
$$

The first term on the right hand side we can write in the equivalent form and get

$$
\begin{aligned}
& |u(0)|^{2}|S| m^{-1} \delta^{m} \\
& \quad \leq 2 \int_{\partial B_{\delta}} d S \int_{0}^{\delta}\left|\int_{0}^{r} \frac{\partial u}{\partial \varrho} \varrho^{\frac{\alpha+m-1}{2}} \varrho^{-\frac{\alpha+m-1}{2}} d \varrho\right|^{2} r^{m-1} d r+2 \int_{B}|u|^{2} d x .
\end{aligned}
$$

Applying the Hölder inequality to the inner integral, we obtain the estimate

$$
\begin{aligned}
& |u(0)|^{2}|S| m^{-1} \\
& \quad \leq \frac{1}{\gamma} \int_{\partial B_{\delta}} d S \int_{0}^{\delta} \int_{0}^{r}|\nabla u|^{2} \varrho^{\alpha+m-1} d \varrho r^{m-1+2 \gamma} d r+2 \int_{B}|u|^{2} d x
\end{aligned}
$$


Putting $\delta$ instead of the upper bound of the inner integral we get the following :

$$
|u(0)|^{2}|S| m^{-1} \delta^{m} \leq \frac{\delta^{m+2 \gamma}}{\gamma(m+2 \gamma)} \int_{B_{\delta}}|\nabla u|^{2} r^{\alpha+m-1} d r+2 \int_{B}|u|^{2} d x .
$$

Dividing by $\delta^{m}$ and taking into account that $m(m+2 \gamma)^{-1}<1$, we obtain the inequality

$$
|u(0)|^{2} \leq \frac{\delta^{2 \gamma}}{\gamma|S|} \int_{B_{\delta}}|\nabla u|^{2} r^{\alpha} d x+\frac{2 m}{|S| \delta^{m}} \int_{B}|u|^{2} d x .
$$

Using the notation (2.6) we obtain the inequalities (2.4) and (2.5).

COROLlaRY 2.1. - Let $\lambda$ be the smallest absolute value of the eigenvalues for the operator $\Delta$ with condition (2.2). Then the inequalities

$$
|u(0)|^{2} \leq \eta \int_{B}|D u|^{2} r^{\alpha} d x+\frac{C_{0}(\eta)}{\lambda^{2}} \int_{B}|\Delta u|^{2} d x
$$

and

$$
\sum_{i}^{m}\left|u_{i}(0)\right|^{2} \leq \eta \int_{B}\left|D^{2} u\right|^{2} r^{\alpha} d x+\frac{C_{0}(\eta)}{\lambda} \int_{B}|\Delta u|^{2} d x
$$

take place if $u$ satisfies (2.2).

Proof. - In fact both of the second terms on the right hand side of (2.4) and (2.5) can be easily estimated by the integral of $|\Delta u|^{2}$.

Using the condition (2.2) and integrating by parts we have

$$
\int_{B}|D u|^{2} d x=-\int_{B} u \Delta u d x \leq\left(\int_{B}|u|^{2} d x\right)^{\frac{1}{2}}\left(\int_{B}|\Delta u|^{2} d x\right)^{\frac{1}{2}} .
$$

Then

$$
\int_{B}|u|^{2} d x \leq \frac{1}{\lambda^{2}} \int_{B}|\Delta u|^{2} d x
$$

and from the previous inequality we have

$$
\int_{B}|D u|^{2} d x \leq \frac{1}{\lambda} \int_{B}|\Delta u|^{2} d x
$$

and so the corollary is proved. 
LeMma 2.2. - For $u \in W_{2, \alpha}^{(2)}(B)$, satisfying (2.2), the equality

$$
\begin{aligned}
& \int_{B} u_{i k} u_{i k} r^{\alpha} d x \\
& =\int_{B}|\Delta u|^{2} r^{\alpha} d x+\alpha \int_{B}\left[u_{i}(x)-u_{i}(0)\right] \\
& \quad \times\left[u_{k k} \cos \left(x_{i}, r\right)-u_{i k} \cos \left(x_{k}, r\right)\right] r^{\alpha-1} d x-(m-1) \int_{\partial B}\left|u_{r}\right|^{2} d S
\end{aligned}
$$

holds, where $u_{r}$ is the derivative with respect to $r$.

Proof. - Integrating twice by parts we have

$$
\begin{aligned}
\int_{B} u_{i k} u_{i k} d x= & \int_{B}\left[u_{i}-u_{i}(0)\right]_{k}\left[u_{i}-u_{i}(0)\right]_{k} d x \\
= & \int_{B}|\Delta u|^{2} d x+\int_{\partial B}\left\{\left[u_{i}-u_{i}(0)\right] u_{i k} \cos \left(r, x_{k}\right)\right. \\
& \left.-\left[u_{i}-u_{i}(0)\right] u_{k k} \cos \left(r, x_{i}\right)\right\} d S
\end{aligned}
$$

Therefore

$$
\begin{aligned}
& \int_{\partial B}\left\{\left[u_{i}-u_{i}(0)\right] u_{i k} \cos \left(r, x_{k}\right)-\left[u_{i}-u_{i}(0)\right] u_{k k} \cos \left(r, x_{i}\right)\right\} d S \\
& \quad=\int_{\partial B}\left(\left|D^{2} u\right|^{2}-|\Delta u|^{2}\right) d x
\end{aligned}
$$

With the same kind of calculations (see for example [3] p. 142 etc.) we come to the identity

$$
\begin{aligned}
\int_{B} u_{i k} u_{i k} r^{\alpha} d x= & \int_{B}|\Delta u|^{2}|x|^{\alpha} d x \\
& +\alpha \int_{B}\left[u_{i}-u_{i}(0)\right] u_{k k} r^{\alpha-1} \cos \left(x_{i}, r\right) d x \\
& -\alpha \int_{B}\left[u_{i}-u_{i}(0)\right] u_{i k} r^{\alpha-1} \cos \left(x_{k}, r\right) d x \\
& +\int_{\partial B}\left\{\left[u_{i}-u_{i}(0)\right] u_{i k} \cos \left(x_{k}, r\right)\right. \\
& \left.-\left[u_{i}-u_{i}(0)\right] \Delta u \cos \left(x_{i}, r\right)\right\} d S
\end{aligned}
$$

Vol. $12, \mathrm{n}^{\circ} 4-1995$. 
After applying (2.10) we arrive at

$$
\begin{aligned}
\int_{B} u_{i k} u_{i k} r^{\alpha} d x= & \int_{B}|\Delta u|^{2} r^{\alpha} d x+\alpha \int_{B}\left[u_{i}-u_{i}(0)\right]\left[u_{k k} \cos \left(x_{i}, r\right)\right. \\
& \left.-u_{i k} \cos \left(x_{k}, r\right)\right] r^{\alpha-1} d x+\int_{B}\left[u_{i k} u_{i k}-(\Delta u)^{2}\right] d x
\end{aligned}
$$

Under condition (2.2) we have from (2.10) that

$$
\int_{B}\left(\left|D^{2} u\right|^{2}-|\Delta u|^{2}\right) d x=-(m-1) \int_{\partial B}\left|u_{r}\right|^{2} d x
$$

and we come to (2.9).

Consider a function

$$
v(x)=u(x)-u(0)-u_{i}(0) x_{i},
$$

which evidently satisfies the conditions

$$
v(0)=v_{i}(0)=0 \quad \text { and } \quad v_{i k}=u_{i k} .
$$

Take a complete orthonormal set of spherical functions

$$
\left\{Y_{j, l}(\theta)\right\} \quad\left(j=0,1,2, \ldots ; l=1, \ldots, k_{j}, \theta \in S\right)
$$

and consider the expansion

$$
v(x)=\sum_{j=0}^{+\infty} \sum_{l=1}^{k_{j}} v_{j, l}(r) Y_{j, l}(\theta) .
$$

The derivatives of $v_{j, l}(r)$ with respect to $r$ we denote by $v_{j, l}^{\prime}(r)$.

LEMMA 2.3. - For any $u \in W_{2, \alpha}^{(2)}(B)$, satisfying (2.2), the identity

$$
\begin{aligned}
\int_{B}\left|D^{2} u\right|^{2} r^{\alpha} d x \\
=\int_{B}|\Delta u|^{2} r^{\alpha} d x-(m-1) \int_{\partial B}\left|u_{r}\right|^{2} d S-\frac{\alpha}{2} \int_{\partial B}|\nabla v|^{2} d S \\
\quad+\frac{\alpha}{2} \int_{\partial B}\left|v_{r}\right|^{2} d S-\frac{\alpha}{2} \sum_{j, l} j(j+m-2) v_{j, e}^{2}(1) \\
\quad+\alpha \sum_{j, l} \int_{0}^{1}\left[(m-1)\left|v_{j, l}^{\prime}\right|^{2}\right. \\
\left.\quad+(\alpha+m-3) j(j+m-2)\left|v_{j, l}\right|^{2} r^{-2}\right] r^{\alpha+m-3} d r
\end{aligned}
$$


holds true (by $\sum_{j, l}$ we understand the summation in the same limits as in (2.12)).

Proof. - We can write the identity (2.9) in the form

$$
\begin{aligned}
\int_{B}\left|D^{2} u\right|^{2} r^{\alpha} d x= & \int_{B}|\Delta u|^{2} r^{\alpha} d x-(m-1) \int_{\partial B}\left|u_{r}\right|^{2} d S \\
& +\alpha \int_{B} v_{r} \Delta v r^{\alpha-1} d x-\alpha \int_{B} v_{i} v_{i r} r^{\alpha-1} d x .
\end{aligned}
$$

Using (2.11) we can integrate by parts in the last term on the right hand side

$$
\begin{aligned}
& \int_{B} v_{i} v_{i r} r^{\alpha-1} d x \\
& =\frac{1}{2} \int_{B}\left(|\nabla v|^{2}\right)_{r} r^{\alpha-1} d x=\frac{1}{2} \int_{\partial B} d S \int_{0}^{1}\left(|\nabla v|^{2}\right)_{r} r^{\alpha+m-2} d r \\
& =\frac{1}{2} \int_{\partial B} d S\left[\left.|\nabla v|^{2} r^{\alpha+m-2}\right|_{0} ^{1}-(\alpha+m-2) \int_{0}^{1}|\nabla v|^{2} r^{\alpha+m-3} d r\right] \\
& =\frac{1}{2} \int_{\partial B}|\nabla v|^{2} d S-\frac{\alpha+m-2}{2} \int_{B}|\nabla v|^{2} r^{\alpha-2} d x .
\end{aligned}
$$

So

$$
\begin{aligned}
& \int_{B}\left|D^{2} u\right|^{2} r^{\alpha} d x \\
& =\int_{B}|\Delta u|^{2} r^{\alpha} d x-(m-1) \int_{\partial B}\left|u_{r}\right|^{2} d S+\alpha \int_{B} v_{r} \Delta v r^{\alpha-1} d x \\
& \quad+\frac{\alpha(\alpha+m-2)}{2} \int_{B}|\nabla v|^{2} r^{\alpha-2} d x-\frac{\alpha}{2} \int_{\partial B}|\nabla v|^{2} d S
\end{aligned}
$$

Integrating by parts we get

$$
\begin{aligned}
& \int_{B}|\nabla v|^{2} r^{\alpha-2} d x \\
&=\int_{B} v_{i} v_{i} r^{\alpha-2} d x=\int_{B}\left(v v_{i} r^{\alpha-2}\right)_{i} d x \\
& \quad-\int_{B} v \Delta v r^{\alpha-2} d x-(\alpha-2) \int_{B} v v_{i} r^{\alpha-3} r_{i} d x \\
&=\int_{\partial B} v v_{r} d S-\int_{B} v \Delta v r^{\alpha-2} d x-(\alpha-2) \\
& \quad \times \int_{\partial B} d S \int_{0}^{1} v v_{r} r^{\alpha+m-4} d r=\int_{\partial B} v v_{r} d S-\frac{\alpha-2}{2} \int_{\partial B}|v|^{2} d S \\
&-\int_{B} v \Delta v r^{\alpha-2} d x+\frac{(\alpha-2)(\alpha+m-4)}{2} \int_{0}^{1}|v|^{2} r^{\alpha-4} d x .
\end{aligned}
$$

Vol. $12, n^{\circ} 4-1995$. 
Finally

$$
\begin{aligned}
\int_{B}|\nabla v|^{2} r^{\alpha-2} d x= & \int_{\partial B}\left(v v_{r}-\frac{\alpha-2}{2}|v|^{2}\right) d S-\int_{B} v \Delta v r^{\alpha-2} d x \\
& +\frac{(\alpha-2)(\alpha+m-4)}{2} \int_{B}|v|^{2} r^{\alpha-4} d x .
\end{aligned}
$$

Substituting in (2.14) we come to

$$
\begin{aligned}
& \int_{B}\left|D^{2} u\right|^{2} r^{\alpha} d x \\
&=\int_{B}|\Delta u|^{2} r^{\alpha} d x-(m-1) \int_{\partial B}\left|u_{r}\right|^{2} d S \\
&-\frac{\alpha}{2} \int_{\partial B}\left[|\nabla v|^{2}-(\alpha+m-2)\left(v v_{r}-\frac{\alpha-2}{2}|v|^{2}\right)\right] d S \\
&+\alpha \int_{B} v_{r} \Delta v r^{\alpha-1} d r-\frac{\alpha(\alpha+m-2)}{2} \int_{B} v \Delta v r^{\alpha-2} d x \\
&+\frac{\alpha(\alpha-2)(\alpha+m-2)(\alpha+m-4)}{4} \int_{B}|v|^{2} r^{\alpha-4} d x
\end{aligned}
$$

Let us transform the last three terms on the right hand side of (2.15) with the help of the expansion (2.12). Then

$$
\begin{aligned}
I_{1}= & \int_{B} v_{r} \Delta v r^{\alpha-1} d x \\
= & \sum_{j, l} \int_{0}^{1} v_{j, l}^{\prime}\left[\left(r^{m-1} v_{j, l}^{\prime}\right)^{\prime} r^{\alpha-m}-j(j+m-2) v_{j, l}^{\prime} v_{j, l} r^{\alpha-3}\right] r^{m-1} d r \\
= & \sum_{j, l}\left\{\left.r^{\alpha+m-2} v_{j, l}^{\prime} v_{j, l}^{\prime}\right|_{0} ^{1}-\int_{0}^{1}\left[v_{j, l}^{\prime \prime} v_{j, l}^{\prime} r^{\alpha+m-2}\right.\right. \\
& \left.\left.+(\alpha-1)\left(v_{j, l}^{\prime}\right)^{2} r^{\alpha+m-3}\right] d r-j(j+m-2) \int_{0}^{1} v_{j, l}^{\prime} v_{j, l} r^{\alpha+m-4} d r\right\} \\
= & \int_{\partial B}\left|v_{r}\right|^{2} d S-\sum_{j, l}\left\{\left.\frac{1}{2}\left(v_{j, l}^{\prime}\right)^{2} r^{\alpha+m-2}\right|_{0} ^{1} \int_{0}^{1}\left|v_{j, l}\right|^{2} r^{\alpha+m-3} d r\right\} \\
& -\frac{(\alpha+m-2)}{2} \int_{0}^{1} v_{j, l}^{\prime} v_{j, l} r^{\alpha+m-4} d r+(\alpha-1) \int_{B}\left|v_{r}\right|^{2} r^{\alpha+m-3} d r
\end{aligned}
$$




$$
\begin{aligned}
= & \frac{1}{2} \int_{\partial B}\left|v_{r}\right|^{2} d S+\frac{m-\alpha}{2} \int_{B}\left|v_{r}\right|^{2} r^{\alpha-2} d x \\
& -\sum_{j, l} j(j+m-2) \int_{B} v_{j, l}^{\prime} v_{j, l} r^{\alpha+m-4} d r \\
= & \frac{1}{2} \int_{\partial B}\left|v_{r}\right|^{2} d S-\frac{1}{2} \sum_{j, l} j(j+m-2) \\
& \times\left|v_{j, l}(1)\right|^{2}+\frac{\alpha+m-4}{2} \sum_{j, l} j(j+m-2) \int_{0}^{1}\left|v_{j, l}\right|^{2} r^{\alpha+m-5} d r \\
& +\frac{m-\alpha}{2} \int_{B}\left|v_{r}\right|^{2} r^{\alpha-2} d x .
\end{aligned}
$$

So

(2.16) $I_{1}=\int_{B} v_{r} \Delta v r^{\alpha-1} d x$

$$
\begin{aligned}
= & \frac{1}{2} \int_{\partial B}\left|v_{r}\right|^{2} d S-\frac{1}{2} \sum_{j, l} j(j+m-2)\left|v_{j, l}(1)\right|^{2} \\
& +\frac{\alpha+m-4}{2} \sum_{j, l} j(j+m-2) \int_{0}^{1}\left|v_{j, l}\right|^{2} r^{\alpha+m-5} d r \\
& +\frac{m-\alpha}{2} \int_{B}\left|v_{r}\right|^{2} r^{\alpha-2} d x .
\end{aligned}
$$

Now

$$
\begin{aligned}
I_{2}= & \int_{B} v \Delta v r^{\alpha-2} d x \\
= & \sum_{j, l} \int_{0}^{1} v_{j, l}\left[v_{j, l}^{\prime \prime}+(m-1) r^{-1} v_{j, l}^{\prime}-j(j+m-2) r^{-2} v_{j, l}\right] r^{\alpha+m-3} d r \\
= & \sum_{j, l}\left[\int_{0}^{1} v_{j, l} v_{j, l}^{\prime \prime} r^{\alpha+m-3} d r+(m-1)\right. \\
& \left.\times \int_{0}^{1} v_{j, l} v_{j, l}^{\prime} r^{\alpha+m-4} d r-j(j+m-2) \int_{0}^{1}\left|v_{j, l}\right|^{2} r^{\alpha+m-5} d r\right]
\end{aligned}
$$

Vol. $12, \mathbf{n}^{\circ} 4-1995$. 


$$
\begin{aligned}
= & \sum_{j, l}\left[\left.v_{j, l} v_{j, l}^{\prime} r^{\alpha+m-3}\right|_{0} ^{1}-\int_{0}^{1}\left|v_{j, l}^{\prime}\right|^{2} r^{\alpha+m-3} d r\right. \\
& -(\alpha+m-3) \int_{0}^{1} v_{j, l} v_{j, l}^{\prime} r^{\alpha+m-4} d r \\
& +\left.\frac{m-1}{2}\left|v_{j, l}\right| r^{\alpha+m-4}\right|_{0} ^{1}-(m-1) \frac{\alpha+m-4}{2} \int_{0}^{1}\left|v_{j, l}\right|^{2} r^{\alpha+m-5} d r \\
& \left.-j(j+m-2) \int_{0}^{1} v_{j, l}^{2} r^{\alpha+m-5} d r\right] .
\end{aligned}
$$

Then

$$
\begin{aligned}
I_{2}= & \int_{B} v \Delta v r^{\alpha-2} d x \\
= & \sum_{j, l}\left\{v_{j, l}(1) v_{j, l}^{\prime}(1)-\frac{\alpha-2}{2}\left|v_{j, l}(1)\right|^{2}\right. \\
& +\left[\frac{(\alpha+m-4)(\alpha-2)}{2}-j(j+m-2)\right] \\
& \times \int_{0}^{1}\left|v_{j, l}\right|^{2} r^{\alpha+m-5} d r-\int_{0}^{1}\left|v_{j, l}^{\prime}\right|^{2} r^{\alpha+m-3} d r .
\end{aligned}
$$

Combining (2.15), (2.16) and (2.17) we get (2.13)

LEMMA 2.4. - If $v \in W_{2, \alpha}^{(2)}(B)$ satisfies (2.2) and vanishes with all first derivatives at the center of $B$ then the identity

$$
\text { (2.18) } \begin{aligned}
\int_{B} \mid \Delta & \left.v\right|^{2} r^{\alpha} d x \\
= & (m-1) \int_{\partial B}\left|v_{r}\right|^{2} d S-2 \sum_{j, l} j(j+m-2) v_{j, l}^{\prime}(1) v_{j, l}(1) \\
& +(\alpha-2) \sum_{j, l} j(j+m-2)\left|v_{j, l}(1)\right|^{2}+\sum_{j, l} \int_{0}^{1}\left\{\left|v_{j, l}^{\prime \prime}\right|^{2}\right. \\
& +[(m-1)(1-\alpha)+2 j(j+m-2)]\left|v_{j, l}^{\prime}\right|^{2} r^{-2}+j(j+m-2) \\
& \left.\times[j(j+m-2)+(2-\alpha)(\alpha+m-4)]\left|v_{j, l}\right|^{2} r^{-4}\right\} r^{\alpha+m-1} d r
\end{aligned}
$$

holds true. 
Proof. - Using the expansion (2.12) we get

$$
\begin{aligned}
& \int_{B}|\Delta v|^{2} r^{\alpha} d x \\
& =\sum_{j, l}\left\{\int_{0}^{1}\left[\left|v_{j, l}^{\prime \prime}\right|^{2}+(m-1)^{2} r^{-2}\left|v_{j, l}^{\prime}\right|^{2}+j^{2}(j+m-2)^{2} r^{-4}\left|v_{j, l}\right|^{2}\right]\right. \\
& \times r^{\alpha+m-1} d r+2(m-1) \int_{0}^{1} v_{j, l}^{\prime \prime} v_{j, l}^{\prime} r^{\alpha+m-2} d r-2 j(j+m-2) \\
& \times \int_{0}^{1} v_{j, l}^{\prime \prime} v_{j, l} r^{\alpha+m-3} d r-2(m-1) j(j+m-2) \\
& \left.\times \int_{0}^{1} v_{j, l}^{\prime} v_{j, l} r^{\alpha+m-4} d r\right\} \\
& =\sum_{j, l}\left\{\int _ { 0 } ^ { 1 } \left[\left|v_{j, l}^{\prime \prime}\right|^{2}+(m-1)^{2}\left|v_{j, l}^{\prime}\right|^{2} r^{-2}+j^{2}(j+m-2)^{2} r^{-4}\right.\right. \\
& \left.\times\left|v_{j, l}\right|^{2}\right] r^{\alpha+m-1} d r+2(m-1)\left[\left.\frac{\left|v^{\prime}\right|^{2}}{2} r^{\alpha+m-2}\right|_{0} ^{1}-\frac{\alpha+m-2}{2}\right. \\
& \left.\times \int_{0}^{1}\left|v_{j, l}^{\prime}\right|^{2} r^{\alpha+m-3} d r\right] \\
& -2 j(j+m-2)\left[\left.v_{j, l}^{\prime} v_{j, l} r^{\alpha+m-3}\right|_{0} ^{1}-\int_{0}^{1}\left|v_{j, l}^{\prime}\right|^{2}\right. \\
& \times r^{\alpha+m-3} d r-(\alpha+m-3) \int_{0}^{1} v_{j, l}^{\prime} v_{j, l} r^{\alpha+m-4} d r \\
& -\frac{2(m-1) j(j+m-2)}{2} \\
& \left.\times\left[\left.\left|v_{j, l}\right|^{2} r^{\alpha+m-4}\right|_{0} ^{1}-(\alpha+m-4) \int_{0}^{1}\left|v_{j, l}\right|^{2} r^{\alpha+m-5} d r\right]\right\} .
\end{aligned}
$$

Continuing this process we come to

$$
\begin{aligned}
& \int_{B}|\Delta v|^{2} r^{\alpha} d x \\
& =\sum_{j, l}\left\{\int_{0}^{1}\left[\left|v_{j, l}^{\prime \prime}\right|^{2}+(m-1)^{2}\left|v_{j, l}^{\prime}\right|^{2} r^{-2}+j^{2}(j+m-2)^{2}\left|v_{j, l}\right|^{2} r^{-4}\right]\right. \\
& \times r^{\alpha+m-1} d r+(m-1)\left|v_{j, l}^{\prime}(1)\right|^{2} \\
& -(m-1)(\alpha+m-2) \int_{0}^{1}\left|v_{j, l}^{\prime}\right|^{2} r^{\alpha+m-3} d r \\
& -2 j(j+m-2) v_{j, l}^{\prime}(1) v_{j, l}(1)+2 j(j+m-2) \int_{0}^{1}\left|v_{j, l}^{\prime}\right|^{2} r^{\alpha+m-3} d r
\end{aligned}
$$




$$
\begin{aligned}
& +2(\alpha+m-3) j(j+m-2) \int_{0}^{1} v_{j, l}^{\prime} v_{j, l} r^{\alpha+m-4} d r \\
& -(m-1) j(j+m-2)\left|v_{j, l}(1)\right|^{2} \\
& \left.+(m-1)(\alpha+m-4) j(j+m-2) \int_{0}^{1}\left|v_{j, l}\right|^{2} r^{\alpha+m-5} d r\right\} .
\end{aligned}
$$

In the same way we get

$$
\begin{aligned}
\int_{B} \mid \Delta & \left.v\right|^{2} r^{\alpha} d x \\
= & \sum_{j, l} \int_{0}^{1}\left[\left|v_{j, l}^{\prime \prime}\right|^{2}+(m-1)^{2}\left|v_{j, l}^{\prime}\right|^{2} r^{-2}+j^{2}(j+m-2)^{2}\left|v_{j, l}\right|^{2} r^{-4}\right] \\
& \times r^{\alpha+m-1} d r+(m-1)\left|v_{j, l}^{\prime}(1)\right|^{2}-(m-1)(\alpha+m-2) \int_{0}^{1}\left|v_{j, l}^{\prime}\right|^{2} \\
& \times r^{\alpha+m-3} d r-2 j(j+m-2) v_{j, l}^{\prime}(1) v_{j, l}(1) \\
& +2 j(j+m-2) \int_{0}^{1}\left|v_{j, l}^{\prime}\right|^{2} \\
& \times r^{\alpha+m-3} d r+2(\alpha+m-3) j(j+m-2)\left[\left.\frac{\left|v_{j, l}\right|^{2}}{2} r^{\alpha+m-4}\right|_{0} ^{1}\right. \\
& \left.-\frac{(\alpha+m-4)}{2} \int_{0}^{1}\left|v_{j, l}\right|^{2} r^{\alpha+m-5} d r\right] \\
& -(m-1) j(j+m-2)\left|v_{j, l}(1)\right|^{2} \\
& +(m-1)(\alpha+m-4) j(j+m-2) \int_{0}^{1}\left|v_{j, l}\right|^{2} r^{\alpha+m-5} d r .
\end{aligned}
$$

After simple calculations we come to (2.18).

LEMMA 2.5. - For any $u \in W_{2, \alpha}^{(2)}(B)$ satisfying (2.2) the inequality

$$
\begin{aligned}
& \int_{B}\left|D^{2} u\right|^{2} r^{\alpha} d x \\
& \leq\left(1+M_{\gamma}^{2}\right) \int_{B}|\Delta u|^{2} r^{\alpha} d x-(m-1) \int_{\partial B}\left|u_{r}\right|^{2} d S \\
& \quad+\frac{(m-2+2 \gamma)(m-1)+m M_{\gamma}^{2}}{m} \sum_{i=1}^{m}\left|u_{i}(0)\right|^{2}|S| \\
& \quad+\left[(m+1+2 \gamma) M_{\gamma}^{2}+\frac{m-2+2 \gamma}{2}\right](m-1)|u(0)|^{2}|S|
\end{aligned}
$$


holds, where

$$
M_{\gamma}^{2}=\frac{\left.(m-2+2 \gamma)\{1+\gamma)^{2}+\left[2-(1-\gamma)^{2}\right] m\right\}}{(m+1+\gamma)^{2}(1-\gamma)^{2}}
$$

Proof. - From (2.18) we have

$$
\begin{aligned}
\sum_{j, l} & \int_{0}^{1}\left\{\left|v_{j, l}^{\prime \prime}\right|^{2}+[(m-1)(1-\alpha)+2 j(j+m-2)]\left|v_{j, l}^{\prime}\right|^{2} r^{-2}\right. \\
& +j(j+m-2) \\
& \left.\times[j(j+m-2)+(2-\alpha)(\alpha+m-4)]\left|v_{j, l}\right|^{2} r^{-4}\right\} r^{\alpha+m-1} d r \\
= & \int_{B}|\Delta v|^{2} r^{\alpha} d x-(m-1) \int_{\partial B}\left|v_{r}^{\prime}\right|^{2} d S \\
& +2 \sum_{j, l} j(j+m-2) v_{j, l}^{\prime}(1) v_{j, l}(1) \\
& -(\alpha-2) \sum_{j, l} j(j+m-2)\left|v_{j, l}(1)\right|^{2} .
\end{aligned}
$$

According to [3] (p. 51 and p. 54) for $\alpha=2-m-2 \gamma(0<\gamma<1)$ we have

$$
\begin{aligned}
& \alpha \sum_{j, l} \int_{0}^{1}\left[(m-1)\left|v_{j, l}^{\prime}\right|^{2}+(\alpha+m-3) j(j+m-2)\left|v_{j, l}\right|^{2} r^{-2}\right] r^{\alpha+m-3} d r \\
& \leq M_{\gamma}^{2} \sum_{j, l} \int_{0}^{1}\left\{\left|v_{j, l}^{\prime \prime}\right|^{2}+[(m-1)(1-\alpha)+2 j(j+m-2)]\left|v_{j, l}^{\prime}\right|^{2} r^{-2}\right. \\
& \quad+j(j+m-2)[j(j+m-2) \\
& \left.\quad+(2-\alpha)(\alpha+m-4)]\left|v_{j, l}\right|^{2} r^{-4}\right\} r^{\alpha+m-1} d r .
\end{aligned}
$$

The fact that in this case $v$ and $\nabla v$ can differ from zero on $\partial B$ plays no role. 
Then from (2.13) and (2.18) we have

$$
\begin{aligned}
\int_{B}\left(\left|D^{2} u\right|-|\Delta u|^{2}\right) r^{\alpha} d x \\
\leq- \\
\quad-(m-1) \int_{\partial B}\left|u_{r}\right|^{2} d S+\frac{\alpha}{2} \int_{\partial B}\left|v_{r}^{\prime}\right|^{2} d S \\
\quad-\frac{\alpha}{2} \int_{\partial B}|\nabla v|^{2} d S+M_{\gamma}^{2}\left[\int_{B}|\Delta v|^{2} r^{\alpha} d x-(m-1) \int_{\partial B}\left|v_{r}\right|^{2} d S\right. \\
\left.\quad+2 \sum_{j, l} j(j+m-2) v_{j, l}^{\prime}(1) v_{j, l}(1)-(\alpha-2) \sum_{j, l} j(j+m-2) v_{j, l}^{2}(1)\right] \\
\quad-\frac{\alpha}{2} \sum_{j, l} j(j+m-2)\left|v_{j, l}(1)\right|^{2} .
\end{aligned}
$$

Since $u$ vanishes on $\partial B$ and, according to (2.11), $v$ is a linear function on $\partial B$ we have that $v_{j, l}=0$ on $\partial B$ for $j>1$. Therefore

$$
\begin{aligned}
& \left|\sum_{j, l} j(j+m-2) v_{j, l}^{\prime}(1) v_{j, l}(1)\right| \\
& \quad=(m-1)\left|\sum_{l=1}^{k_{1}} v_{1, l}^{\prime}(1) v_{1, l}(1)\right| \\
& \quad \leq\left(\sum_{l=1}^{k_{1}}\left|v_{1, l}^{\prime}(1)\right|^{2}\right)^{\frac{1}{2}}\left(\sum_{l=1}^{k_{1}}\left|v_{1, l}(1)\right|^{2}\right)^{\frac{1}{2}}(m-1) \\
& \quad \leq\left(\int_{\partial B}\left|v_{r}\right|^{2} d S\right)^{\frac{1}{2}}\left(\int_{\partial B}|v|^{2} d S\right)^{\frac{1}{2}}(m-1)
\end{aligned}
$$

So

$$
\begin{aligned}
& \left|\sum_{j, l} j(j+m-2) v_{j, l}^{\prime}(1) v_{j, l}(1)\right| \\
& \quad \leq(m-1)\left(\int_{\partial B}\left|v_{r}\right|^{2} d S\right)^{\frac{1}{2}}\left(\int_{\partial B}|v|^{2} d S\right)^{\frac{1}{2}}
\end{aligned}
$$

In the same way we come to the inequality

$$
\sum_{j, l} j(j+m-2)\left|v_{j, l}(1)\right|^{2} \leq(m-1) \int_{\partial B}|v|^{2} d S .
$$


With the help of (2.21) and (2.22) we get

$$
\begin{aligned}
& \int_{B}\left|D^{2} u\right|^{2} r^{\alpha} d x \\
& \leq\left(1+M_{\gamma}^{2}\right) \int_{B}|\Delta v|^{2} r^{\alpha} d x-(m-1) \int_{\partial B}\left|u_{r}\right|^{2} d S \\
&-\frac{\alpha}{2} \int_{\partial B}|\nabla v|^{2} d S+\frac{\alpha}{2} \int_{\partial B}\left|v_{r}\right|^{2} d S \\
&+(m-1) M_{\gamma}^{2}\left[-\int_{\partial B}\left|v_{r}\right|^{2} d S+2\left(\int_{\partial B}\left|v_{r}\right|^{2} d S\right)^{\frac{1}{2}}\right. \\
&\left.\times\left(\int_{\partial B}|v|^{2} d S\right)^{\frac{1}{2}}-(\alpha-2) \int_{\partial B}|v|^{2} d S\right] \\
&-\frac{\alpha}{2}(m-1) \int_{\partial B}|v|^{2} d S .
\end{aligned}
$$

Let us estimate now the right-hand side of (2.23). Evidently from (2.11) we have

$$
\begin{aligned}
\frac{\alpha}{2} \int_{\partial B}\left(\left|v_{r}\right|^{2}-|\nabla v|^{2}\right) d S & \\
= & \frac{\alpha}{2} \int_{\partial B}\left\{\left|u_{r}^{\prime}-\left(\sum_{i=1}^{m} u_{i}(0) x_{i}\right)_{r}^{\prime}\right|^{2}-\sum_{i=1}^{m}\left|u_{i}-u_{i}(0)\right|^{2}\right\} d S \\
= & \frac{\alpha}{2} \int_{\partial B}\left[\left|u_{r}\right|^{2}-2 u_{r} \sum_{i=1}^{m} u_{i}(0) \cos \left(r, x_{i}\right)+\left(\sum_{i=1}^{m} u_{i}(0) \cos \left(r, x_{i}\right)\right)^{2}\right. \\
& \left.-\sum_{i=1}^{m}\left|u_{i}\right|^{2}+2 \sum_{i=1}^{m} u_{i} u_{i}(0)-\sum_{i=1}^{m}\left|u_{i}(0)\right|^{2}\right] d S .
\end{aligned}
$$

Taking into account that on $\partial B|\nabla u|^{2}=u_{r}^{2}$ and $u_{i}=u_{r} \cos \left(r, x_{i}\right)$, after cancelling some terms we come to the equality

$$
\begin{aligned}
\frac{\alpha}{2} \int_{\partial B}\left(\left|v_{r}\right|^{2}-|\nabla v|^{2}\right) d S= & \frac{\alpha}{2} \int_{\partial B}\left\{\sum_{i=1}^{m}\left|u_{i}(0)\right|^{2}\left[\cos ^{2}\left(r, x_{i}\right)-1\right]\right. \\
& \left.+2 \sum_{i<k} u_{i}(0) u_{k}(0) \cos \left(r, x_{i}\right) \cos \left(r, x_{k}\right)\right\} d S .
\end{aligned}
$$

After easy calculations we have

$$
\frac{\alpha}{2} \int_{\partial B}\left(\left|v_{r}\right|^{2}-|\nabla v|^{2}\right) d S=\frac{\alpha(1-m)}{2 m} \sum_{i=1}^{m}\left|u_{i}(0)\right|^{2}|S| .
$$


Applying the inequality

$$
2 a b<a^{2}+b^{2}
$$

to the middle term in quadratic brackets on the right-hand side of (2.23) and taking into account that $\alpha-3=-(m+1+2 \gamma)$, we obtain

$$
\begin{aligned}
& \int_{B}\left|D^{2} u\right| r^{\alpha} d x \\
& \leq\left(1+M_{\gamma}^{2}\right) \int_{B}|\Delta u|^{2} r^{\alpha} d x-(m-1) \int_{\partial B}\left|u_{r}\right|^{2} d S \\
&+\frac{(m-2+2 \gamma)(m-1)}{2 m} \sum_{i=1}^{m}\left|u_{i}(0)\right|^{2}|S| \\
&+(m+1+2 \gamma)(m-1) M_{\gamma}^{2} \int_{\partial B}|v|^{2} d S \\
&-\alpha \frac{(m-1)}{2} \int_{\partial B}|v|^{2} d S .
\end{aligned}
$$

Since

$$
\int_{\partial B}|v|^{2} d S=u^{2}(0)|S|+\sum_{i=1}^{m} u_{i}^{2}(0)|S| m^{-1}
$$

we come to (2.19).

Lemma 2.6. - If $u \in W_{2, \alpha}^{(2)}(B)$ satisfies (2.2), then for any $\eta>0$ the inequality

$$
\begin{aligned}
\int_{B}\left|D^{2} u\right|^{2} r^{\alpha} d x & \\
& \times\left\{1-\eta|S|\left[\frac{(m-2+2 \gamma)(m-1)+m M_{\gamma}^{2}}{m}\right.\right. \\
& \left.\left.+\frac{2(m+1+2 \gamma) M_{\gamma}^{2}+m-2+2 \gamma}{(1-\gamma)^{2}}(m-1)\right]\right\} \\
\leq & \left(1+M_{\gamma}^{2}\right) \int_{B}|\Delta u|^{2} r^{\alpha} d x+C_{0}(\eta)|S| \\
& \times\left\{\frac{(m-2+2 \gamma)(m-1)+m M_{\gamma}^{2}}{m} \times \int_{B}|\nabla u|^{2} d x\right. \\
& +(m-1)\left[(m+1+2 \gamma) M_{\gamma}^{2}\right. \\
& \left.+(m-2+2 \gamma) / 2] \int_{B}|u|^{2} d x\right\}+(m-1) \\
& \times\left[|S| \frac{(m+1+2 \gamma) M_{\gamma}^{2}+(m-2+2 \gamma) / 2}{1-\gamma} \eta-1\right] \int_{\partial B}\left|u_{r}^{\prime}\right|^{2} d S
\end{aligned}
$$


takes place. Here $\alpha=2-m-2 \gamma(0<\gamma<1), C_{0}(\eta)$ and $M_{\gamma}^{2}$ are determined by (2.6) and (2.20). The value $|S|$ (the area of the unit sphere in $R^{m}$ ) is determined by the formula

$$
|S|=2 \pi^{\frac{m}{2}} / \Gamma\left(\frac{m}{2}\right)
$$

Proof. - From the identity

$$
u_{i}=\left.u_{i}\right|_{r=1}+\int_{1}^{r}\left(u_{i}\right)_{\rho}^{\prime} d \rho
$$

follows the inequality

$$
\int_{B}\left|u_{i}\right|^{2} r^{\alpha} d x \leq\left. 2 \int_{B}\left|u_{i}\right|_{r=1}\right|^{2} r^{\alpha} d x+2 \int_{B}\left|\int_{1}^{r} u_{i \rho} d \rho\right|^{2} r^{\alpha} d x .
$$

Evidently

$$
\left.\int_{B}\left|u_{i}\right|_{r=1}\right|^{2} r^{\alpha} d x=\left.\frac{1}{2(1-\gamma)} \int_{\partial B}\left|u_{i}\right|_{r=1}\right|^{2} d S
$$

Since $u$ satisfies the condition (2.2) we have

$$
\left.\sum_{i=1}^{m} \int_{B}\left|u_{i}\right|_{r=1}\right|^{2} r^{\alpha} d x=\frac{1}{2(1-\gamma)} \int_{\partial B}\left|u_{r}\right|^{2} d S
$$

From the Hardy inequality follows

$$
\int_{B}\left|\int_{1}^{r} u_{i \rho} d \rho\right|^{2} r^{\alpha} d x \leq \frac{1}{(1-\gamma)^{2}} \int_{B}\left|u_{i r}\right|^{2} r^{\alpha+2} d x
$$

So, taking into account that $r \leq 1$, we come to the inequality

$$
\sum_{i=1}^{m} \int_{B}\left|\int_{1}^{r} u_{i \rho} d \rho\right|^{2} r^{\alpha} d x \leq \frac{1}{(1-\gamma)^{2}} \int_{B}\left|D^{2} u\right|^{2} r^{\alpha} d x .
$$

Applying (2.26) and (2.27) we get

$$
\int_{B}|D u|^{2} r^{\alpha} d x \leq \frac{1}{1-\gamma} \int_{\partial B}\left|u_{r}\right|^{2} d S+\frac{2}{(1-\gamma)^{2}} \int_{B}\left|D^{2} u\right|^{2} r^{\alpha} d x
$$

Now combining (2.4), (2.5), (2.19) and (2.28) we come to the inequality (2.25). 
COROLlary 2.2. - We can also apply the inequalities (2.4) and (2.5). Then we arrive at the relation

$$
\begin{aligned}
\int_{B} \mid & \left.D^{2} u\right|^{2} r^{\alpha} d x\left\{1-\eta|S|\left[\frac{(m-2+2 \gamma)(m-1)+m M_{\gamma}^{2}}{m}\right.\right. \\
& \left.\left.+\frac{2(m+1+2 \gamma) M_{\gamma}^{2}+m-2+2 \gamma}{(1-\gamma)^{2}}(m-1)\right]\right\} \\
\leq & \left(1+M_{\gamma}^{2}\right) \int_{B}|\Delta u|^{2} r^{\alpha} d x+\frac{C_{0}(\eta)|S|}{\lambda} \\
& \times\left\{\frac{(m-2+2 \gamma)(m-1)+m M_{\gamma}^{2}}{m}\right. \\
& \left.+\frac{(m-1)\left[(m+1+2 \gamma) M_{\gamma}^{2}+(m-2+2 \gamma) / 2\right]}{\lambda}\right\} \\
& \times \int_{B}|\Delta u|^{2} d x+(m-1) \\
& \times\left[|S| \frac{(m+1+2 \gamma) M_{\gamma}^{2}+(m-2+2 \gamma) / 2}{1-\gamma} \eta-1\right] \int_{\partial B}\left|u_{r}\right|^{2} d S
\end{aligned}
$$

COROLlaRY 2.3. - Taking into account that $r \leq 1$, we can write

$$
\int_{B}|\Delta u|^{2} d x \leq \int_{B}|\Delta u|^{2} r^{\alpha} d x
$$

and from (2.29) then follows

$$
\begin{aligned}
\int_{B} \mid & \left.D^{2} u\right|^{2} r^{\alpha} d x\left\{1-\eta|S|\left[\frac{(m-2+2 \gamma)(m-1)+m M_{\gamma}^{2}}{m}\right.\right. \\
& \left.\left.+\frac{2(m+1+2 \gamma) M_{\gamma}^{2}+m-2+2 \gamma}{(1-\gamma)^{2}}(m-1)\right]\right\} \\
\leq & \left(1+M_{\gamma}^{2}+\frac{C_{0}(\eta)|S|}{\lambda}\left\{\frac{(m-2+2 \gamma)(m-1)+m M_{\gamma}^{2}}{m}\right.\right. \\
& \left.\left.+\frac{(m-1)\left[(m+1+2 \gamma) M_{\gamma}^{2}+(m-2+2 \gamma) / 2\right]}{\lambda}\right\}\right) \\
& \times \int_{B}|\Delta u|^{2} r^{\alpha} d x+(m-1) \\
& \times\left[|S| \frac{(m+1+2 \gamma) M_{\gamma}^{2}+(m-2+2 \gamma) / 2}{1-\gamma} \eta-1\right] \int_{\partial B}\left|u_{r}\right|^{2} d S
\end{aligned}
$$


It is easy to see, that if

$$
\begin{aligned}
1- & \eta|S|\left[\frac{(m-2+2 \gamma)(m-1)+m M_{\gamma}^{2}}{m}\right. \\
& \left.+\frac{2(m+1+2 \gamma) M_{\gamma}^{2}+m-2+2 \gamma}{(1-\gamma)^{2}}(m-1)\right]
\end{aligned}
$$

is nonnegative, then the expression

$$
|S| \frac{(m+1+2 \gamma) M_{\gamma}^{2}+(m-2+2 \gamma) / 2}{1-\gamma} \eta-1
$$

is nonpositive. After rescaling in $x$ we come to the following

THEOREM 2.1. - Let $u \in W_{2, \alpha}^{(2)}\left(B_{R}\right)$ satisfies the condition (2.2). Let also the inequality

$$
\begin{aligned}
E \equiv & 1-\eta|S|\left[\frac{(m-2+2 \gamma)(m-1)+m M_{\gamma}^{2}}{m}\right. \\
& \left.+\frac{2(m+1+2 \gamma) M_{\gamma}^{2}+m-2+2 \gamma}{(1-\gamma)^{2}}(m-1)\right]>0
\end{aligned}
$$

holds. Then the following estimates

$$
\begin{aligned}
& \int_{B_{R}}\left|D^{2} u\right|^{2} r^{\alpha} d x \\
& \leq \frac{1}{E}\left\{\left(1+M_{\gamma}^{2}\right) \int_{B_{R}}|\Delta u|^{2} r^{\alpha} d x+C_{0}(\eta)|S|\right. \\
& \times\left[\frac{(m-2+2 \gamma)(m-1)+m M_{\gamma}^{2}}{m} R^{\alpha-2} \int_{B_{R}}|\nabla u|^{2} d x\right. \\
&+(m-1)\left[(m+1+2 \gamma) M_{\gamma}^{2}\right. \\
&\left.\left.+(m-2+2 \gamma) / 2] R^{\alpha-4} \int_{B_{R}}|u|^{2} d x\right]\right\}
\end{aligned}
$$

$$
\begin{aligned}
& \int_{B_{R}}\left|D^{2} u\right|^{2} r^{\alpha} d x \\
& \quad \leq \frac{1}{E}\left\{\left(1+M_{\gamma}^{2}\right) \int_{B_{R}}|\Delta u|^{2} r^{\alpha} d x+\frac{C_{0}(\eta)|S|}{\lambda} R^{\alpha}\right.
\end{aligned}
$$

Vol. $12, \mathrm{n}^{\circ}$ 4-1995. 


$$
\begin{aligned}
& \times\left[\frac{(m-2+2 \gamma)(m-1)+m M_{\gamma}^{2}}{m}\right. \\
& \left.+\frac{(m-1)\left((m+1+2 \gamma) M_{\gamma}^{2}+(m-2+2 \gamma) / 2\right)}{\lambda}\right] \\
& \left.\times \int_{B_{R}}|\Delta u|^{2} d x\right\}
\end{aligned}
$$

and

$$
\begin{aligned}
& \int_{B_{R}}\left|D^{2} u\right|^{2} r^{\alpha} d x \\
& \leq \frac{1}{E}\left\{1+M_{\gamma}^{2}+\frac{C_{0}(\eta)|S|}{\lambda}\left[\frac{(m-2+2 \gamma)(m-1)+m M_{\gamma}^{2}}{m}\right.\right. \\
&\left.\left.+\frac{(m-1)\left((m+1+2 \gamma) M_{\gamma}^{2}+(m-2+2 \gamma) / 2\right)}{\lambda}\right]\right\} \\
& \times \int_{B_{R}}^{-}|\Delta u|^{2} r^{\alpha} d x
\end{aligned}
$$

hold true.

Let us recall that $\alpha=2-m-2 \gamma(0<\gamma<1), M_{\gamma}$ and $C_{0}(\eta)$ are defined by (2.20) and (2.6) respectively, $\lambda$ is the least absolute value of the eigenvalues for the operator $\Delta$ in $B$ with condition (2.2) and

$$
|S|=2 \pi^{m / 2} / \Gamma(m / 2)
$$

is the area of the unit sphere in $R^{m}$.

Consider now the cylinder $Q_{R}=(0, T) \times B_{R}\left(Q_{1}=Q\right)$ with boundary conditions

$$
\left.u\right|_{\partial B_{R}}=\left.u\right|_{t=0}=0
$$

for a function $u(t, x)$ given in $Q$. Denote $\beta=-\alpha$ and omit for a while the index $R$.

For $m>2$ the inequality

$$
\int_{Q}|\Delta u|^{2} r^{\beta} d x d t \leq \frac{m}{m-\beta} \int_{Q}\left|\varepsilon \partial_{t} u-\Delta u\right|^{2} r^{\beta} d x d t
$$

was proven in [4] ( $\varepsilon$ is an arbitrary nonnegative value). 
Lemma 2.7. - Let $m=2$ and therefore $\beta=2 \gamma(0<\gamma<1)$. Suppose that $u$ satisfies (2.36) and $u \in L_{2}\left\{(0, T) ; W_{2, \beta}^{(2)}(B)\right\}$. Then the inequality

$$
\begin{aligned}
& \int_{Q}|\Delta u|^{2} r^{\beta} d x d t \\
& \quad \leq\left[1+\frac{\beta}{2-\beta}+\frac{2-\beta}{\left(1-\frac{\beta}{4}\right)^{2} \beta}\right] \int_{Q}\left|\varepsilon \partial_{t} u-\Delta u\right|^{2} r^{\beta} d x d t
\end{aligned}
$$

holds, where $\varepsilon$ is an arbitrary nonnegative constant.

Proof. - Denote

$$
\varepsilon \dot{u}-\Delta u=f
$$

multiply this equality by $\Delta u \cdot r^{\beta}$ and integrate by parts on the left-hand side. Then according to lemma 2 in our paper ([2], (2.38)) we get

$$
\begin{aligned}
& \left.\frac{\varepsilon}{2} \int_{B}|\nabla u|^{2} r^{\beta} d x\right|_{t=T}+\beta \int_{Q} \Delta u u_{r}^{\prime} d x d t+\int_{B}|\Delta u|^{2} r^{\beta} d x d t \\
& \quad=\int_{Q} f\left(\Delta u+\beta u_{r}^{\prime} r^{-1}\right) r^{\beta} d x d t
\end{aligned}
$$

After using for $u(x, t)$ the expansion, analogous to (2.12), according to the same lemma in [2] $((2.39))$, we come to the inequality

$$
\begin{aligned}
\int_{Q}|\Delta u|^{2} r^{\beta} d x d t+\frac{\beta(2-\beta)}{2} \sum_{s, k} \int_{0}^{T} \int_{0}^{1}\left|u_{s, k}^{\prime}\right|^{2} r^{\beta-1} d r d t \\
\leq \int_{Q} f\left(\Delta u+\beta u_{r}^{\prime} r^{-1}\right) r^{\beta} d x d t \\
\quad+\frac{\beta(2-\beta)}{2} \sum_{s, k} s^{2} \int_{0}^{T} \int_{0}^{1}\left|u_{s, k}\right|^{2} r^{\beta-3} d r
\end{aligned}
$$

Now we have to estimate the right-hand side term of (2.40). As in [2] we multiply (2.39) by $u_{s, k} r^{\beta-2}(s \geq 1)$. Integrating by parts we come to the inequality ([2], (3.30) etc.)

$$
\left[\left(1-\frac{\beta}{4}\right) \beta+s^{2}-1\right] \int_{0}^{T} \int_{0}^{1}\left|u_{s, k}\right|^{2} r^{\beta-3} d r d t \leq\left|\int_{0}^{T} \int_{0}^{1} f_{s, k} u_{s, k} r^{\beta-1} d r d t\right|
$$

Vol. 12, $n^{\circ} 4-1995$. 
It is clear that

$$
\begin{aligned}
& \sum_{s \geq 1} s^{2} \int_{0}^{T} \int_{0}^{1}\left|u_{s, k}\right|^{2} r^{\beta-3} d r d t \\
& \quad \leq \frac{1}{\left(1-\frac{\beta}{4}\right) \beta} \sum_{s \geq 1}\left[\left(1-\frac{\beta}{4}\right) \beta+s^{2}-1\right] \int_{0}^{T} \int_{0}^{1}\left|u_{s, k}\right|^{2} r^{\beta-3} d r d t .
\end{aligned}
$$

Thus we have

$$
\sum_{s \geq 1} s^{2} \int_{0}^{T} \int_{0}^{1}\left|u_{s, k}\right|^{2} r^{\beta-3} d r d t \leq \frac{1}{\left(1-\frac{\beta}{4}\right) \beta}\left|\int_{0}^{T} \int_{0}^{1} f_{s, k} u_{s, k} r^{\beta-1} d r d t\right|
$$

After applying the Hölder's inequality we get

$$
\sum_{s \geq 1} s^{2} \int_{0}^{T} \int_{0}^{1}\left|u_{s, k}\right|^{2} r^{\beta-3} d r d t \leq \frac{1}{\left(1-\frac{\beta}{4}\right)^{2} \beta^{2}} \int_{Q}|f|^{2} r^{\beta} d x .
$$

With the help of (2.40) we come to the inequality

$$
\begin{aligned}
& \int_{Q}|\Delta u|^{2} r^{\beta} d x d t+\frac{\beta(2-\beta)}{2} \sum_{s, k} \int_{0}^{T} \int_{0}^{1}\left|u_{s, k}^{\prime}\right|^{2} r^{\beta-1} d r d t \\
& \leq \frac{2-\beta}{2\left(1-\frac{\beta}{4}\right)^{2} \beta} \int_{Q}|f|^{2} r^{\beta} d x d t+\int_{Q} f\left(\Delta u+\beta u_{r}^{\prime} r^{-1}\right) r^{\beta} d x d t
\end{aligned}
$$

Applying well known inequalities we get

$$
\begin{aligned}
& \int_{Q} f\left(\Delta u+\beta u_{r}^{\prime} r^{-1}\right) r^{\beta} d x d t \\
& \quad \leq\left|\int_{Q} f \Delta u r^{\beta} d x d t\right|+\eta \beta \int_{Q}\left|u_{r}^{\prime}\right|^{2} r^{\beta-2} d x d t+\frac{\beta}{4 \eta} \int_{Q}|f|^{2} r^{\beta} d x d t .
\end{aligned}
$$

According to expansion (2.12)

$$
\int_{Q}\left|u_{r}^{\prime}\right|^{2} r^{\beta-2} d x d t=\sum_{s, k} \int_{0}^{T} \int_{0}^{1}\left|u_{s, k}^{\prime}\right|^{2} r^{\beta-1} d r d t
$$


and we can write

$$
\begin{aligned}
& \left|\int_{Q} f\left(\Delta u+\beta u_{r}^{\prime} r^{-1}\right) r^{\beta} d x d t \leq\right| \int_{Q} f \Delta u r^{\beta} d x d t \mid \\
& \quad+\eta \beta \sum_{s, k} \int_{0}^{T} \int_{0}^{1}\left|u_{s, k}^{\prime}\right|^{2} r^{\beta-1} d r d t+\frac{\beta}{4 \eta} \int_{Q}|f|^{2} r^{\beta} d x d t .
\end{aligned}
$$

So, from (2.41) we come to

$$
\begin{array}{r}
\int_{Q}|\Delta u|^{2} r^{\beta} d x d t+\frac{\beta(2-\beta)}{2} \sum_{s, k} \int_{0}^{T} \int_{0}^{1}\left|u_{s, k}^{\prime}\right|^{2} r^{\beta-1} d r d t \\
\leq\left|\int_{Q} f \Delta u r^{\beta} d x d t\right|+\eta \beta \sum_{s, k} \int_{0}^{T} \int_{0}^{1}\left|u_{s, k}^{\prime}\right|^{2} r^{\beta-1} d r d t \\
+\frac{\beta}{4 \eta} \int_{Q}|f|^{2} r^{\beta} d x d t+\frac{2-\beta}{2\left(1-\frac{\beta}{4}\right)^{2}} \int_{Q}|f|^{2} r^{\beta} d x d t
\end{array}
$$

Taking $\eta=\frac{2-\beta}{2}$ and applying the inequality

$$
\left|\int_{Q} f \Delta u r^{\beta} d x d t\right| \leq \frac{1}{2} \int_{Q}|f|^{2} r^{\beta} d x d t+\frac{1}{2} \int_{Q}|\Delta u|^{2} r^{\beta} d x d t
$$

we get (2.38).

Set

$$
A_{\alpha, m}^{2}= \begin{cases}1-\frac{\alpha}{2+\alpha}-\frac{2+\alpha}{\left(1+\frac{\alpha}{4}\right)^{2} \alpha} & m=2 \\ \frac{m}{m+\alpha} & m>2 .\end{cases}
$$

THEOREM 2.2. - Suppose $u \in L_{2}\left\{(0, T) ; W_{2, \alpha}^{(2)}\left(B_{R}\right)\right\}$ and satisfies the boundary conditions (2.36), $\alpha \in(-m,-2-m) \cup(3-m, 0)$ and $\gamma=(2-m-\alpha) / 2$. Then the following estimates

$$
\begin{aligned}
& \int_{Q_{R}}\left|D^{2} u\right|^{2} r^{\alpha} \zeta d x d t \\
& \leq \frac{1}{E}\left\{A_{\alpha, m}^{2}\left(1+M_{\gamma}^{2}\right) \int_{Q_{R}}\left|\varepsilon \partial_{t} u-\Delta u\right|^{2} r^{\alpha} \zeta d x d t+C_{0}(\eta)|S|\right. \\
& \quad \times\left[\frac{(m-2+2 \gamma)(m-1)+m M_{\gamma}^{2}}{m} R^{\alpha-2} \int_{Q_{R}}|\nabla u|^{2} d x d t\right.
\end{aligned}
$$

Vol. $12, \mathrm{n}^{\circ}$ 4-1995. 


$$
\begin{aligned}
& +(m-1)\left[(m+1+2 \gamma) M_{\gamma}^{2}+(m-2+2 \gamma) / 2\right] R^{\alpha-4} \\
& \left.\left.\times \int_{Q_{R}}|u|^{2} d x d t\right]\right\}+C R^{\alpha} \int_{Q_{R}}\left|\varepsilon \partial_{t} u-\Delta u\right|^{2} d x d t
\end{aligned}
$$

$$
\begin{aligned}
\int_{Q_{R}} & \left|D^{2} u\right|^{2} r^{\alpha} \zeta d x d t \\
\leq & \frac{1}{E}\left\{A_{\alpha, m}^{2}\left(1+M_{\gamma}^{2}\right) \int_{Q_{R}}|\varepsilon \dot{u}-\Delta u|^{2} r^{\alpha} \zeta d x d t+\frac{C_{0}(\eta)|S|}{\lambda} R^{\alpha}\right. \\
& \times\left[\frac{(m-2+2 \gamma)(m-1)+m M_{\gamma}^{2}}{m}\right. \\
& \left.+\frac{(m-1)\left((m-1+2 \gamma) M_{\gamma}^{2}+(m-2+2 \gamma) / 2\right)}{\lambda}\right] \\
& \left.\times \int_{Q_{R}}|\Delta u|^{2} d x d t\right\}+C R^{\alpha} \int_{Q_{R}}|\varepsilon \dot{u}-\Delta u|^{2} d x d t
\end{aligned}
$$

and

$$
\begin{aligned}
\int_{Q_{R}} \mid & \left.D^{2} u\right|^{2} r^{\alpha} \zeta d x d t \\
\leq & \frac{1}{E}\left\{A_{\alpha, m}^{2}\left(1+M_{\gamma}^{2}\right)+\frac{C_{0}(\eta)|S|}{\lambda}\right. \\
& \times\left[\frac{(m-2+2 \gamma)(m-1)+m M_{\gamma}^{2}}{m}\right. \\
& \left.\left.+\frac{(m-1)\left((m+1+2 \gamma) M_{\gamma}^{2}+(m-2+2 \gamma) / 2\right)}{\lambda}\right]\right\} \\
& \times \int_{Q_{R}}|\varepsilon \dot{u}-\Delta u|^{2} r^{\alpha} \zeta d x d t+C R^{\alpha} \int_{Q_{R}}|\varepsilon \dot{u}-\Delta u|^{2} d x d t
\end{aligned}
$$

hold where $\varepsilon$ and $T$ are arbitrary positive values, and all other constants are defined at the end of the formulation of theorem (2.1) and by (2.42) $(C$ does not depend on $\varepsilon$ and $R$ ).

The function $\zeta$ is a smooth monotone cut-off function, defined by the relation

$$
\zeta(r)=\left\{\begin{array}{cl}
1 & 0 \leq r \leq \frac{1}{2} R \\
\text { smooth } & \frac{1}{2} R \leq r \leq \frac{3}{4} R \\
0 & \frac{3}{4} R \leq r .
\end{array}\right.
$$


Proof. - We can assume at first that $u$ is as smooth as we wish. Let $w(t, x)$ be a solution in $Q$ of the following boundary value problem

$$
\begin{gathered}
\varepsilon \partial_{t} w+\Delta w=-\Delta u r^{\alpha} \zeta \\
\left.w\right|_{t=T}=\left.w\right|_{\partial B_{R}}=0 .
\end{gathered}
$$

Multiply equation (2.47) by $\Delta u \zeta$ and integrate once by parts with respect to $t$ and twice with respect to $x$. Then we get

$$
\int_{Q}\left(\varepsilon \partial_{t} u-\Delta u\right) \Delta w \zeta d x d t=\int_{Q}|\Delta u|^{2} r^{\alpha} \zeta^{2} d x d t+\ldots
$$

where the nonwritten terms are those containing the derivatives of $\zeta$. Applying the Hölder inequality, we get

$$
\begin{aligned}
& \left(\int_{Q}|\Delta u|^{2} r^{\alpha} \zeta^{2} d x d t\right)^{2} \\
& \quad \leq\left(\int_{Q}\left|\varepsilon \partial_{t} u-\Delta u\right|^{2} r^{\alpha} \zeta^{2} d x d t\right)^{\frac{1}{2}}\left(\int_{Q}|\Delta w|^{2} r^{-\alpha} d x d t\right)^{\frac{1}{2}} \\
& \quad+C \int_{Q}\left|\varepsilon \partial_{t} u-\Delta u\right|^{2} d x d t .
\end{aligned}
$$

It is trivial that $w$ also satisfies the inequalities (2.37) and (2.38) (one only has to exchange $t$ by $T-t$ ). Therefore

$$
\begin{aligned}
\int_{Q}|\Delta w|^{2} r^{-\alpha} d x d t & \leq A_{\alpha, m}^{2} \int_{Q}\left|\varepsilon \partial_{t} w+\Delta w\right|^{2} r^{-\alpha} d x d t \\
& =A_{\alpha, m}^{2} \int_{Q}|\Delta u|^{2} r^{\alpha} \zeta^{2} d x d t .
\end{aligned}
$$

Now from (2.48) after rescaling we get the results of the theorem, if we take into account that

$$
\int_{Q}|\Delta u|^{2} r^{\alpha} d x d t \geq \int_{Q}|\Delta u|^{2} r^{\alpha} \zeta^{2} d x d t-C \int_{Q}\left|\varepsilon \partial_{t} u-\Delta u\right|^{2} d x d t .
$$

Let us return now to inequalities (2.4) and (2.5) of lemma (2.1). Since the power of the integrals on the right-hand side of these inequalities is equal to one they belong to the so-called class of the linear inequalities. However Vol. $12, n^{\circ} 4-1995$. 
in some problems it is important to have the so-called multiplicative inequalities. We shall obtain them in the following.

LEMMA 2.8. - If $u \in W_{2, \alpha}^{(2)}\left(B_{R}\right)$ and (2.2) takes place, then the inequalities

$$
\begin{aligned}
|u(0)|^{2} & \leq C\left(\int_{B_{R}}|D u|^{2} r^{\alpha} d x\right)^{\frac{m}{m+2 \gamma}}\left(\int_{B_{R}}|u|^{2} d x\right)^{\frac{2 \gamma}{m+2 \gamma}} \\
\sum_{i=0}^{m}\left|u_{i}(0)\right|^{2} & \leq C\left(\int_{B}\left|D^{2} u\right|^{2} r^{\alpha} d x\right)^{\frac{m}{m+2 \gamma}}\left(\int_{B}|D u|^{2} d x\right)^{\frac{2 \gamma}{m+2 \gamma}}
\end{aligned}
$$

hold.

Proof. - Evidently it is enough to prove only the first of the inequalities (2.49). Substituting in (2.4) the expression (2.6), we get

$$
|u(0)|^{2}<\eta \int_{B_{R}}|D u|^{2} r^{\alpha} d x+C \eta^{-\frac{m}{2 \gamma}} \int_{B_{R}}|u|^{2} d x .
$$

Take now

$$
\eta=\left(\int_{B_{R}}|D u|^{2} r^{\alpha} d x\right)^{-2 \gamma /(m+2 \gamma)}\left(\int_{B_{R}}|u|^{2} d x\right)^{2 \gamma /(m+2 \gamma)}
$$

and we come to (2.49) (if $|D u|=0$ then $u \equiv 0$ and (2.49) is trivial.)

Remark 2.1. - Under the assumption of the lemma the inequality

$$
|u(0)|^{2} \leq C\left(\int_{B_{R}}\left|D^{2} u\right|^{2} r^{\alpha} d x\right)^{\frac{m}{m+2 \gamma}}\left(\int_{B_{R}}|D u|^{2} d x\right)^{\frac{2 \gamma}{m+2 \gamma}}
$$

holds.

In fact

$$
\begin{aligned}
\int_{B}|D u|^{2} r^{\alpha} d x & \leq\left. 2 \int_{B_{R}}|D u-D u|_{0}\right|^{2} r^{\alpha} d x+\left.2 \int_{B_{R}}|D u|_{0}\right|^{2} r^{\alpha} d x \\
& \leq C\left[\left.\int_{B_{R}}|D u-D u|_{0}\right|^{2} r^{\alpha-2} d x+\left.|D u|_{0}\right|^{2}\right] .
\end{aligned}
$$

From the well known Hardy inequality and from (2.5) it follows that

$$
\int_{B_{R}}|D u|^{2} r^{\alpha} d x \leq C\left(\int_{B_{R}}\left|D^{2} u\right|^{2} r^{\alpha} d x+\int_{B_{R}}|D u|^{2} d x\right) .
$$


Then from (2.2) and inequalities $r \leq 1$ and $\alpha<0$ we get

$$
\begin{aligned}
\int_{B}|D u|^{2} r^{\alpha} d x & \leq C\left(\int_{B_{R}}\left|D^{2} u\right|^{2} r^{\alpha} d x+\int_{B_{R}}\left|D^{2} u\right|^{2} d x\right) \\
& \leq C \int_{B_{R}}\left|D^{2} u\right|^{2} r^{\alpha} d x .
\end{aligned}
$$

Applying (2.49) and (2.50) to (2.19) we come to

THEOREM 2.3. - Let $u \in W_{2, \alpha}^{(2)}\left(B_{R}\right)$ and satisfy (2.2). Then the inequality

$$
\begin{aligned}
& \int_{B_{R}}\left|D^{2} u\right|^{2} r^{\alpha} d x \\
& \leq\left(1+M_{\gamma}^{2}\right) \int_{B_{R}}|\Delta u|^{2} r^{\alpha} d x+C\left(\int_{B_{R}}\left|D^{2} u\right|^{2} r^{\alpha} d x\right)^{\frac{m}{m+2 \gamma}} \\
& \quad \times\left(\int_{B_{R}}|D u|^{2} d x\right)^{\frac{2 \gamma}{m+2 \gamma}}
\end{aligned}
$$

holds true.

Proof. - In fact we omit on the right-hand side of (2.19) the negative term and apply (2.49) and (2.50). Then we get

$$
\begin{aligned}
\int_{B_{R}}\left|D^{2} u\right|^{2} r^{\alpha} d x & \\
\leq & \left(1+M_{\gamma}^{2}\right) \int_{B_{R}}|\Delta u|^{2} r^{\alpha} d x+C\left[\left(\int_{B_{R}}\left|D^{2} u\right|^{2} r^{\alpha} d x\right)^{\frac{m}{m+2 \gamma}}\right. \\
& \left.+\left(\int_{B_{R}}|D u|^{2} r^{\alpha} d x\right)^{\frac{m}{m+2 \gamma}}\right]\left(\int_{B_{R}}|D u|^{2} d x\right)^{\frac{2 \gamma}{m+2 \gamma}} .
\end{aligned}
$$

After using the relation

$$
\int_{B_{R}}|u|^{2} d x \leq \int_{B_{R}}|D u|^{2} d x
$$

we come to the result.

Suppose now that condition (2.2) is not satisfied; how will estimate (2.51) change in this case? 
THEOREM 2.4. - Let $u \in W_{2, \alpha}^{(2)}\left(B_{R}\right)$. Then the inequality

$$
\begin{aligned}
& \int_{B_{R}}\left|D^{2} u\right|^{2} r^{\alpha} \zeta d x \\
& \leq\left(1+M_{\gamma}^{2}+\eta\right) \int_{B_{R}}|\Delta u|^{2} r^{\alpha} \zeta d x \\
& \quad+C\left\{\left(\int_{B_{R}}\left|D^{2} u\right|^{2} r^{\alpha} \zeta d x\right)^{\frac{m}{m+2 \gamma}}\left[\int_{B_{R}}\left(|D u|^{2}+|u|^{2}\right) d x\right]^{\frac{2 \gamma}{m+2 \gamma}}\right. \\
& \left.\quad+\int_{B_{R}}\left(|D u|^{2}+|u|^{2}\right) d x\right\}
\end{aligned}
$$

holds true, where $\zeta$ is defined by (2.46) and $\eta$ is an arbitrary small positive number.

The result follows immediately by substituting the function $u \zeta$ for $u$ in (2.51).

THEOREM 2.5. - Suppose that $u \in L_{2}\left\{(0, T) ; W_{2, \alpha}^{(2)}\left(B_{R}\right)\right\}$ satisfies only the second of the conditions (2.36), $u=0$, when $t=0$. Then the estimate

$$
\begin{aligned}
& \int_{Q_{R}}\left|D^{2} u\right|^{2} r^{\alpha} \zeta d x d t \\
& \leq\left(1+M_{\gamma}^{2}+\eta\right) A_{\alpha, m}^{2} \int_{Q_{R}}\left|\varepsilon \partial_{t} u-\Delta u\right|^{2} r^{\alpha} \zeta d x d t \\
&+C\left\{\left(\int_{Q_{R}}\left|D^{2} u\right|^{2} r^{\alpha} \zeta d x d t\right)^{\frac{m}{m+2 \gamma}}\right. \\
& \times\left[\int_{Q_{R}}\left(|D u|^{2}+|u|^{2}\right) d x d t\right]^{\frac{2 \gamma}{m+2 \gamma}} \\
&\left.+\int_{Q_{R}}\left(|D u|^{2}+|u|^{2}\right) d x d t\right\}
\end{aligned}
$$

holds, where $C$ does not depend on $\varepsilon$.

Proof. - Take a function $w$ which satisfies the equation (2.47) with the same conditions and multiply both sides of the differential equation by $\Delta u \cdot \zeta$. After integration over $B_{R}$ we come to

$$
\int_{Q_{R}}\left(\varepsilon \partial_{t} w+\Delta w\right) \Delta u \zeta d x d t=-\int_{Q_{R}}|\Delta u|^{2} r^{\alpha} \zeta^{2} d x d t
$$


After two integrations by parts on the left-hand side with respect to $x$, we get

$$
\begin{aligned}
\int_{Q_{R}} & {\left[\varepsilon \Delta \partial_{t} w \zeta u+\Delta w \Delta u \zeta\right] d x d t } \\
= & \int_{Q_{R}}|\Delta u|^{2} r^{\alpha} \zeta^{2} d x d t \\
& -2 \varepsilon \int_{Q_{R}} \nabla \partial_{t} w \nabla \zeta \cdot u d x d t-\varepsilon \int_{Q_{R}} \partial_{t} w \Delta \zeta u d x d t
\end{aligned}
$$

Integrating on the left-hand side by parts once with respect to $t$ and on the right-hand side in the second integral once with respect to $x$, we get

$$
\begin{aligned}
\int_{Q_{R}} & \left(\varepsilon \partial_{t} u-\Delta u\right) \Delta w \zeta d x d t \\
= & \int_{Q_{R}}|\Delta u|^{2} r^{\alpha} \zeta^{2} d x d t \\
& +\varepsilon \int_{Q_{R}} \partial_{t} w u \cdot \Delta \zeta d x d t-\varepsilon \int_{Q_{R}} \partial_{t} w \nabla \zeta \nabla u d x d t
\end{aligned}
$$

Therefore

$$
\begin{aligned}
\int_{Q_{R}}|\Delta u|^{2} r^{\alpha} \zeta^{2} d x d t \\
=\int_{Q_{R}}\left(\varepsilon \partial_{t} u-\Delta u\right) \Delta w \zeta d x d t \\
\quad-\int_{Q_{R}}\left(\varepsilon \partial_{t} w-\Delta w\right) u \Delta \zeta d x d t+\int_{Q_{R}}\left(\varepsilon \partial_{t} w-\Delta w\right) \nabla \zeta \nabla u d x d t \\
\quad-\int_{Q_{R}} u \Delta w \Delta \zeta d x d t+\int_{Q_{R}} \Delta w \nabla u \cdot \nabla \zeta d x d t
\end{aligned}
$$

Let us now estimate the integrals on the right-hand side. After applying an elementary inequality we come to the following relations:

$$
\text { 1) } \begin{aligned}
\left|\int_{Q_{R}}\left(\varepsilon \partial_{t} u-\Delta u\right) \Delta w \zeta d x d t\right| \leq & \frac{1}{4 \eta} \int_{Q_{R}}\left|\varepsilon \partial_{t} u-\Delta u\right|^{2} r^{\alpha} \zeta^{2} d x d t \\
& +\eta \int_{Q_{R}}|\Delta w|^{2} r^{-\alpha} d x d t
\end{aligned}
$$




$$
\text { 2) } \begin{aligned}
&\left|\int_{Q_{R}}\left(\varepsilon \partial_{t} w-\Delta w\right) u \Delta \zeta d x d t\right| \\
& \leq \eta \int_{Q_{R}}\left|\varepsilon \partial_{t} w-\Delta w\right|^{2} r^{-\alpha} d x d t \\
&+\frac{1}{4 \eta} \int_{Q_{R}}|u|^{2}|\Delta \zeta|^{2} r^{\alpha} d x d t \\
& \leq \eta_{1} \int_{Q_{R}}|\Delta u|^{2} r^{\alpha} \zeta^{2} d x d t \\
&+C \int_{Q_{R}}|u|^{2} d x d t\left(\eta_{1}>0-\text { arbitrary }\right)
\end{aligned}
$$

3) $\left|\int_{Q_{R}}\left(\varepsilon \partial_{t} w-\Delta w\right) \nabla \zeta \nabla u d x d t\right|$

$$
\begin{aligned}
\leq & \eta_{2} \int_{Q_{R}}|\Delta u|^{2} r^{\alpha} \zeta^{2} d x d t+ \\
& +C \int_{Q_{R}}|\nabla u|^{2} d x d t\left(\eta_{2}>0-\text { arbitrary }\right)
\end{aligned}
$$

Then by (2.37) we get

$$
\begin{aligned}
& \int_{Q_{R}}|\Delta u|^{2} r^{\alpha} \zeta^{2} d x d t \\
& \quad \leq \eta A_{\alpha, m}^{2} \int_{Q_{R}}|\Delta u|^{2} r^{\alpha} \zeta^{2} d x d t \\
& \quad+\frac{1}{4 \eta} \int_{Q_{R}}\left|\varepsilon \partial_{t} u-\Delta u\right|^{2} r^{\alpha} \zeta^{2} d x d t+\eta_{1} \int_{Q_{R}}|\Delta u|^{2} r^{\alpha} \zeta^{2} d x d t \\
& \quad+C \int_{Q_{R}}\left(|D u|^{2}+|u|^{2}\right) d x d t
\end{aligned}
$$

Taking $\eta=A_{\alpha, m}^{2} /(2 m)$ we obtain the inequality

$$
\begin{aligned}
\int_{Q_{R}}|\Delta u|^{2} r^{\alpha} \zeta^{2} d x d t \leq & \left(A_{\alpha, m}^{2}+\eta\right) \int_{Q_{R}}\left|\varepsilon \partial_{t} u-\Delta u\right|^{2} r^{\alpha} \zeta^{2} d x d t \\
& +C \int_{Q_{R}}\left(|D u|^{2}+|u|^{2}\right) d x d t
\end{aligned}
$$

After using Theorem 2.4. the proof of the theorem is concluded. 


\section{COERCIVITY ESTIMATES FOR THE STOKES SYSTEM IN WEIGHTED SPACES}

Consider now at first the stationary Stokes system

$$
\left\{\begin{array}{r}
\Delta u+\nabla p=f \\
\operatorname{div} u=0
\end{array}\right.
$$

with the boundary condition

$$
\left.u\right|_{\partial \Omega}=0 .
$$

We assume that the mean value of $p$ is equal to zero. Using the inequalities (2.52) and (2.53) we shall derive some estimates with explicit constants for the solution of the problem (3.1), (3.2). The Stokes system was very extensively discussed in many books and papers we refer here only to the paper of V. Solonnikov [6] and monograph of O. Ladyzhenskaya [10]. From the results of these paper and monograph in particular follows that if $f \in W_{q}^{(k)}(\Omega)(q>1)$ then the second derivatives of $u$ and the first derivatives of $p$ also belong to this space. The analogous result for the nonstationary system is also included there.

Suppose that $\Omega \subset R^{m}$ is a bounded domain and $\partial \Omega$ is sufficiently smooth.

THEOREM 3.1. - If $f \in L_{2, \alpha}(\Omega)$ with $\alpha=2-m-2 \gamma(0<\gamma<1)$ then the weak solutions of system (3.1) with the boundary condition (3.2) satisfy the inequalities

$$
\begin{aligned}
& \int_{B_{R}}|\nabla p|^{2} r^{\alpha} \zeta d x \\
& \leq {\left[1-\frac{\alpha(m-2)}{m-1}+\eta\right]\left[1-\frac{\alpha(\alpha+m-2)}{2(m-1)}\right]^{-2} } \\
& \times \int_{B_{R}}|f|^{2} r^{\alpha} \zeta d x+C\left(\int_{B_{R}}|p|^{2} d x+\int_{B_{R}}|f|^{2} d x\right)
\end{aligned}
$$

$$
\begin{aligned}
& \int_{B_{R}}\left|D^{2} u\right|^{2} r^{\alpha} \zeta d x \\
& \quad \leq\left\{1+\left[1-\frac{\alpha(m-2)}{m-1}\right]^{\frac{1}{2}}\left[1-\frac{\alpha(\alpha+m-2)}{2(m-1)}\right]^{-1}+\eta\right\}^{2}
\end{aligned}
$$




$$
\begin{aligned}
& \times\left(1+M_{\gamma}^{2}\right) \int_{B_{R}}|f|^{2} r^{\alpha} \zeta d x \\
& +C\left[\left(\int_{B_{R}}\left|D^{2} u\right|^{2} r^{\alpha} \zeta d x\right)^{\frac{m}{m+2 \gamma}}\left(\int_{B_{R}}|D u|^{2} d x\right)^{\frac{2 \gamma}{m+2 \gamma}}\right. \\
& \left.+\int_{B_{R}}|p|^{2} d x+\int_{B_{R}}\left(|D u|^{2}+|u|^{2}\right) d x+\int_{B_{R}}|f|^{2} d x\right]
\end{aligned}
$$

where $x_{0}$ is an arbitrary point inside $\Omega, R<\operatorname{dist}\left(x_{0}, \partial \Omega\right), \eta=$ const $>0$ is arbitrary, $M_{\gamma}$ is defined by (2.20) and $1-2^{-1} \alpha(\alpha+m-2)(m-1)^{-1}>0$.

The proof of this theorem is analogous to the proof which was given in [3] for the solution of the Poisson equation. Let us sketch this proof. According to the above mentioned results of V. Solonnikov we can at first assume that both $f$ and the solution $u, p$ are as smooth as we wish. Take a point $x_{0} \in \Omega$ and consider a ball $B_{R}\left(x_{0}\right)$ with $R<\operatorname{dist}\left(x_{0}, \partial \Omega\right)$. After rescaling we can consider only the ball $B_{1}(0)=B$.

Let $Y_{s, k}(\Theta)(\Theta \in S)$ be a complete orthonormal set of spherical functions and let

$$
p(x)=\sum_{s=0}^{+q \infty} \sum_{k=1}^{k s} p_{s, k}(r) Y_{s, k}(\Theta)
$$

Construct the function

$$
v(x)=\sum_{s=0}^{+\infty} \sum_{k=1}^{k s} v_{s, k}(r) Y_{s, k}(\Theta),
$$

where

$$
\begin{aligned}
v_{0}(r) & =-\int_{r}^{1} p_{0,1}^{\prime}(\varrho) \varrho^{\alpha} d \varrho \\
v_{s, k}(r) & =p_{s, k}(r) r^{\alpha} \quad(s \geq 1) .
\end{aligned}
$$

Take the function

$$
w(x)=v(x) \zeta(r)
$$

where the cut-off function $\zeta(r)$ is determined by (2.46).

Multiplying the Stokes system (3.1) by $\nabla w$ and taking into account that

$$
\int_{B} \Delta u \nabla w d x=-\int_{B} \Delta(d i v u) w d x=0
$$


we come to the equality

$$
\int_{B} \nabla p \nabla w d x=\int_{B} f \nabla w d x
$$

Integrating by parts and substituting the expansions (3.5), (3.6) for $p$ and $w$ we have

$$
\begin{aligned}
\int_{B} & \nabla p \nabla w d x \\
= & \int_{0}^{1}{p^{\prime}}_{0,1}^{2} r^{\alpha+m-1} d r+\sum_{s \geq 1, k} \int_{0}^{1}\left\{\left|p_{s, k}^{\prime}\right|^{2}+[s(s+m-2)\right. \\
& \left.\left.-\frac{\alpha(\alpha+m-2)}{2}\right]\left|p_{s, k}\right|^{2} r^{-2}\right\} r^{\alpha+m-1} \zeta d r+\ldots
\end{aligned}
$$

where the unwritten terms contain only integrals without singularity.

From this immediately follows

$$
\begin{aligned}
\int_{B} & \nabla p \nabla w d x \\
\geq & \int_{0}^{1}\left|p_{0}^{\prime}\right|^{2} r^{\alpha+m-1} d r+\sum_{s \geq 1, k} \int_{0}^{1}\left[\left|p_{s, k}^{\prime}\right|^{2}+s(s+m-2)\right. \\
& \left.\quad \times \min _{s \geq 1} \frac{s(s+m-2)-\alpha(\alpha+m-2) / 2}{s(s+m-2)}\left|p_{s, k}\right|^{2} r^{-2}\right] r^{\alpha+m-1} \zeta d r+\ldots
\end{aligned}
$$

Finally

$$
\int_{B} \nabla p \nabla w d x \geq\left[1-\frac{\alpha(\alpha+m-2) / 2}{m-1}\right] \int_{B}|\nabla p|^{2} r^{\alpha} \zeta d x-c \int_{B}|p|^{2} d x .
$$

On the other hand

$$
\begin{aligned}
\int_{B} \nabla p \nabla w d x & =\int_{B} f \nabla w d x \\
& \leq\left(\int_{B}|f|^{2} r^{\alpha} \zeta d x\right)^{1 / 2}\left(\int_{B_{3 / 4}}|\nabla w|^{2} r^{-\alpha} \zeta^{-1} d x\right)^{1 / 2} .
\end{aligned}
$$

Comparing the last two relations we come to the inequality

$$
\begin{aligned}
& {\left[1-\frac{\alpha(\alpha+m-2)}{2(m-1)}\right] \int_{B}|\nabla p|^{2} r^{\alpha} \zeta d x} \\
& \leq\left(\int_{B}|f|^{2} r^{\alpha} \zeta d x\right)^{1 / 2}\left(\int_{B_{3 / 4}}|\nabla w|^{2} r^{-\alpha} \zeta^{-1} d x\right)^{1 / 2} \\
& \quad+C \int_{\Omega}|p|^{2} d x
\end{aligned}
$$

Vol. 12, $\mathrm{n}^{\circ}$ 4-1995. 
In our book ([3], p. 120 see also [9]) by the same method it was shown that

$$
\begin{aligned}
\int_{\Omega}|\nabla w|^{2} r^{-\alpha} \zeta^{-1} d x \leq & {[1} \\
& \left.-\frac{\alpha(m-2)}{m-1}\right] \int_{\Omega}|\nabla p|^{2} r^{\alpha} \zeta d x \\
& +C \int_{\Omega}|p|^{2} d x .
\end{aligned}
$$

Therefore one of the statements of the theorem is proved.

Take now in the Stokes system (3.1) $\nabla p$ to the right hand side and apply the inequality (2.52). After small calculations you come to the inequality (3.4).

Consider now the nonstationary Stokes system

$$
\left\{\begin{array}{c}
\partial_{t} u-\nu \Delta u+\nabla p=f \quad(\nu=\text { const. }>0) \\
\operatorname{div} u=0
\end{array}\right.
$$

with boundary conditions

$$
\left.u\right|_{\partial \Omega}=\left.u\right|_{t=0}=0
$$

At first we consider the inner estimates.

Suppose $f \in L_{2}\left\{(0, T) ; L_{2, \alpha}(\Omega)\right\}$ and $Q_{R}=(0, T) \times B_{R}$, where $R<\operatorname{dist}\left(x_{0}, \partial \Omega\right)$. It is trivial that estimate (3.3) holds if we change $B_{R}$ for $Q_{R}$.

Then, dividing the first equation of (3.8) by $\nu$ and applying (2.53), we come to

THEOREM 3.2. - Let the conditions for $\alpha$ in theorem 3.1 hold. The solution of the problem (3.8), (3.9) satisfies the inequalities

$$
\begin{aligned}
\int_{Q_{R}} & |\nabla p|^{2} r^{\alpha} \zeta d x d t \\
\leq & {\left[1-\frac{\alpha(m-2)}{m-1}+\eta\right]\left[1-\frac{\alpha(\alpha+m-2)}{2(m-1)}\right]^{-2} } \\
& \times \int_{Q_{R}}|p|^{2} r^{\alpha} \zeta d x d t+C\left(\int_{Q_{R}}|p|^{2} d x d t+\int_{Q_{R}}|f|^{2} d x d t\right)
\end{aligned}
$$

(3.11) $\int_{Q_{R}}\left|D^{2} u\right|^{2} r^{\alpha} \zeta d x d t$

$$
\leq \frac{1}{\nu^{2}}\left\{1+\left[1-\frac{\alpha(m-2)}{m-1}\right]^{\frac{1}{2}}\left[1-\frac{\alpha(\alpha+m-2)}{2(m-1)}\right]^{-1}+\eta\right\}^{2}
$$




$$
\begin{aligned}
& \times\left(1+M_{\gamma}^{2}\right) A_{\alpha, m}^{2} \int_{Q_{R}}|f|^{2} r^{\alpha} \zeta d x d t+C\left[\int_{Q_{R}}|p|^{2} d x d t\right. \\
& +\left(\int_{Q_{R}}\left|D^{2} u\right|^{2} r^{\alpha} \zeta d x d t\right)^{\frac{m}{m+2 \gamma}}\left(\int_{Q_{R}}|D u|^{2} d x d t\right)^{\frac{2 \gamma}{m+2 \gamma}} \\
& \left.+\int_{Q_{R}}\left(|D u|^{2}+|u|^{2}\right) d x d t+\int_{Q_{R}}|f|^{2} d x d t\right]
\end{aligned}
$$

Here $C$ does not depend on $\nu, M_{\gamma}$ and $A_{\alpha, m}$ are relatively defined by (2.20) and (2.42).

For small $\gamma>0$ we have

THEOREM 3.3. - Let the conditions of theorem 3.1. be satisfied, and assume that $\gamma>0$ is small. Then the following estimates for the solutions of system (3.1)

$$
\begin{aligned}
\int_{B_{R}}|\nabla p|^{2} r^{\alpha} \zeta d x \leq & {\left[1+{\left.\frac{(m-2)^{2}}{m-1}+0(\gamma)\right] \int_{B_{R}}|f|^{2} r^{\alpha} \zeta d x}+C\left(\int_{B_{R}}|p|^{2} d x+\int_{B_{R}}|f|^{2} d x d t\right)\right.}
\end{aligned}
$$

$$
\begin{aligned}
& \int_{B_{R}}\left|D^{2} u\right|^{2} r^{\alpha} \zeta d x \\
& \leq \frac{1}{\nu^{2}}\left\{1+\left[1+\frac{(m-2)^{2}}{m-1}+0(\gamma)\right]^{1 / 2}\right\}^{2} \\
& \times\left(1+\frac{m-2}{m+1}\right) \int_{B_{R}}|f|^{2} r^{\alpha} \zeta d x \\
&+C\left[\left(\int_{B_{R}}\left|D^{2} u\right|^{2} r^{\alpha} \zeta d x\right)^{\frac{m}{m+2 \gamma}}\left(\int_{B_{R}}|D u|^{2} d x\right)^{\frac{2 \gamma}{m+2 \gamma}}\right. \\
&\left.+\int_{B_{R}}\left(|D u|^{2}+|u|^{2}\right) d x+\int_{B_{R}}|f|^{2} d x\right]
\end{aligned}
$$

are true.

THEOREM 3.4. - Let the conditions of theorem 3.2 be satisfied, and let $\gamma>0$ be small. Then the inner following estimates for the solutions of the Vol. $12, n^{\circ} 4-1995$. 
system (3.8)

$$
\begin{aligned}
\int_{B_{R}} & |\nabla p|^{2} r^{\alpha} \zeta d x d t \\
\leq & {\left[1+\frac{(m-2)}{m-1}^{2}+0(\gamma)\right] \int_{Q_{R}}|f|^{2} r^{\alpha} \zeta d x d t } \\
& +C \int_{Q_{R}}|f|^{2} d x d t
\end{aligned}
$$

$$
\begin{aligned}
& \int_{Q_{R}}\left|D^{2} u\right|^{2} r^{\alpha} \zeta d x d t \\
& \leq \frac{1}{\nu^{2} \gamma}[2+0(\gamma)] \int_{Q_{R}}|f|^{2} r^{\alpha} \zeta d x d t \\
&+C\left[\int_{Q_{R}}|f|^{2} d x d t+\left(\int_{Q_{R}}\left|D^{2} u\right|^{2} r^{\alpha} \zeta d x d t\right)^{\frac{1}{1+\gamma}}\right. \\
&\left.\times\left(\int_{Q_{R}}|D u|^{2} d x d t\right)^{\frac{\gamma}{1+\gamma}}+\int_{Q_{R}}\left(|D u|^{2}+|u|^{2}\right) d x d t\right],(m=2)
\end{aligned}
$$

$$
\begin{aligned}
\int_{Q_{R}} & \left|D^{2} u\right|^{2} r^{\alpha} \zeta d x d t \\
\leq & \frac{m}{2 \nu^{2}}\left\{1+\left[1+\frac{(m-2)^{2}}{m-1}+0(\gamma)\right]^{1 / 2}\right\} \\
& \times\left(1+\frac{m-2}{m+1}\right) \int_{Q_{R}}|f|^{2} r^{\alpha} \zeta d x d t \\
& +C\left[\int_{Q_{R}}|f|^{2} d x d t+\left(\int_{Q_{R}}\left|D^{2} u\right|^{2} r^{\alpha} \zeta d x d t\right)^{\frac{m}{m+2 \gamma}}\right. \\
& \left.\times\left(\int_{Q_{R}}|D u|^{2} d x d t\right)^{\frac{2 \gamma}{m+2 \gamma}}+\int_{Q_{R}}\left(|D u|^{2}+|u|^{2}\right) d x d t\right]
\end{aligned}
$$

hold true, where $C$ doesn't depend on $\nu$.

It is necessary now for the solution of the problem (3.1) (3.2), to get the estimates in the neighbourhood of the boundary $\partial \Omega$. For this purpose suppose that a piece of the boundary is flat and has the equation $x_{m}=0$. Thus in the neighbourhood the domain $\Omega$ lies in the half space $x_{m}<0$. Take a point $M_{0}\left(x_{1}^{(0)}, \ldots, x_{m}^{(0)}\right)$ in $\Omega$ and consider the ball $B_{R_{0}}\left(M_{0}\right)$ such that

$$
R_{0}>\left|x_{m}^{(0)}\right|
$$


Consider also a parallelelepiped $\Pi_{-}$

$$
\left\{\begin{aligned}
x_{k}^{(0)}-R \leq x_{k} \leq x_{k}^{(0)}+R, & (k=1, \ldots, m-1), \\
x_{m}^{(0)}-R \leq x_{m} \leq 0, & \left(R>R_{0}\right) .
\end{aligned}\right.
$$

Suppose for a moment that $x_{k}^{(0)}=0(k=1, \ldots, m-1)$ and $R=\pi$. The principal part of the estimates doesn't depend on these assumptions. We can suppose at first that all the functions $f, p$ and $u$ are smooth. Expand $f(x)$ in $\Pi$ in the following Fourier series

$$
\left\{\begin{aligned}
f^{(k)}(x)= & \sum_{n} f_{n}^{(k)}\left(x_{m}\right) \cos n_{1} x_{1} \ldots \cos n_{k-1} x_{k-1} \\
& \times \sin n_{k} x_{k} \cos n_{k+1} x_{k+1} \ldots \cos n_{m-1} x_{m-1} \\
& (1 \leq k \leq m-1) \\
f^{(m)}(x)= & \sum_{n} f_{n}^{(m)}\left(x_{m}\right) \cos n_{1} x_{1} \ldots \cos n_{m-1} x_{m-1}
\end{aligned}\right.
$$

where $n=\left(n_{1}, \ldots, n_{m-1},\right)$ and all $n_{k}$ are nonnegative integers. Take

$$
p(x)=\sum_{n} p_{n}\left(x_{m}\right) \cos n_{1} x_{1} \ldots \cos n_{m-1} x_{m-1},
$$

$$
\left\{\begin{array}{l}
u^{(k)}(x)=\sum_{n} u_{n}^{(k)}\left(x_{m}\right) \cos n_{1} x_{1} \ldots \cos n_{k-1} x_{k-1} \\
\times \sin n_{k} x_{k} \cos n_{k+1} x_{k+1} \ldots \cos n_{m-1} x_{m-1}, \\
u^{(m)}(x)=\sum_{n} u_{n}^{(m)}\left(x_{m}\right) \cos n_{1} x_{1} \ldots \cos n_{m-1} x_{m-1} .
\end{array}\right.
$$

Let

$$
\left.u\right|_{x_{m}=0}=0
$$

Then

$$
u_{n}^{(k)}(0)=0, \quad(k=1, \ldots, m) .
$$

We see that the functions $u^{(k)}(x)(k<m)$ satisfy also the following boundary conditions

$$
\left.u^{(k)}\right|_{x_{k}=0, \pi}=0,\left.\quad u_{x_{j}}^{(k)}\right|_{x_{j}=0, \pi}=0 \quad(j \neq k, j \neq m) .
$$


The functions $u(x), p(x)$ satisfy the Stokes system (3.1) in $\Pi^{-}$if the following equalities

$$
\left\{\begin{array}{c}
\ddot{u}_{n}^{(k)}-|n|^{2} u_{n}^{(k)}+n_{k} p_{n}=f_{n}^{(k)}, \quad(k=1, \ldots, m-1), \\
\ddot{u}_{n}^{(m)}-|n|^{2} u_{n}^{(m)}+\dot{p}_{n}=f_{n}^{(m)} \\
\dot{u}_{n}^{(m)}-\sum_{s=1}^{m-1} n_{s} u_{n}^{(s)}=0
\end{array}\right.
$$

where

$$
|n|^{2}=\sum_{s=1}^{m-1} n_{s}^{2}
$$

and dots over $u_{n}^{(k)}$ denote derivatives with respect to $x_{m}$. Multiply the first $m-1$ equations of (3.23) respectively by $n_{k}$. After summation and using the last equation (3.23) we have

$$
\dddot{u}_{n}^{(m)}-|n|^{2} \dot{u}_{n}^{(m)}+|n|^{2} p_{n}=-\sum_{k=1}^{m-1} n_{k} f_{n}^{(k)} .
$$

If we differentiate the second equation of (3.23) with respect to $x_{m}$ and subtract the relation (3.24) from the result we shall have

$$
\ddot{p}_{n}-|n|^{2} p_{n}=\dot{f}_{n}^{(m)}+\sum_{k=1}^{m-1} n_{k} f_{n}^{(k)} \equiv F_{n}^{-}\left(x_{m}\right) .
$$

The bounded solution of this equation for $x_{m}<0$ is given by

$$
\begin{aligned}
p_{n}= & p_{n}^{-}\left(x_{m}\right)=-\frac{1}{2|n|} \int_{-\infty}^{x_{m}} F_{n}^{-}\left(\xi_{m}\right) e^{|n|\left(\xi_{m}-x_{m}\right)} d \xi_{m} \\
& +\frac{1}{2|n|} \int_{0}^{x_{m}} F_{n}^{-}\left(\xi_{m}\right) e^{|n|\left(x_{m}-\xi_{m}\right)} d \xi_{m}+C_{-} e^{|n| x_{m}} .
\end{aligned}
$$

Here $F_{n}^{-}\left(x_{m}\right)$ is a function which coincides with $F_{n}\left(x_{m}\right)$ on $x_{m}>-\pi$ and is continuously expanded on $x_{m}<-\pi$. We suppose also that all the functions are absolutely summable on $(-\infty, 0]$.

Let us also consider the equation (3.25) in $x_{m}>0$ with such suitable right-hand side $F_{n}^{+}\left(x_{m}\right)$ that $p_{n}\left(x_{m}\right)$ is continuous and absolutely summable on the whole strip $-\infty<x_{m}<+\infty$. The solution for $x_{m}>0$ is

$$
\begin{aligned}
p_{n}= & p_{n}^{+}\left(x_{m}\right)=-\frac{1}{2|n|} \int_{0}^{x_{m}} F_{n}^{+}\left(\xi_{m}\right) e^{|n|\left(\xi_{m}-x_{m}\right)} d \xi_{m} \\
& +\frac{1}{2|n|} \int_{+\infty}^{x_{m}} F_{n}^{+}\left(\xi_{m}\right) e^{|n|\left(x_{m}-\xi_{m}\right)} d \xi_{m}+C_{+} e^{-|n| x_{m}}
\end{aligned}
$$


Substitute $x_{m}=0$ in (3.26) and (3.27). Suppose that $C_{-}=C_{+}$and

$$
\int_{-\infty}^{0} F_{n}^{-}\left(\xi_{m}\right) e^{|n| \xi_{m}} d \xi_{m}=\int_{0}^{+\infty} F_{n}^{+}\left(\xi_{m}\right) e^{-|n| \xi_{m}} d \xi_{m} .
$$

Then

$$
p_{n}^{-}(0)=p_{n}^{+}(0) \text {. }
$$

We see that $F_{n}^{-}$should be extended symmetrically on $x_{m}>0$. According to (3.25) the functions $f_{n}^{(m)}\left(x_{m}\right)$ and $f_{n}^{(k)}\left(x_{m}\right)(k=1, \ldots, m-1)$ should be extended respectively in an antisymmetric and symmetric ways. Integrating once by parts we come to

$$
\begin{aligned}
p_{n}= & p_{n}^{-}\left(x_{m}\right)=\frac{1}{2} \int_{-\infty}^{x_{m}} f_{n}^{(m)}\left(\xi_{m}\right) e^{|n|\left(\xi_{m}-x_{m}\right)} d \xi_{m} \\
& +\frac{1}{2} \int_{0}^{x_{m}} f_{n}^{(m)}\left(\xi_{m}\right) e^{|n|\left(x_{m}-\xi_{m}\right)} d \xi_{m} \\
& +\frac{1}{2|n|} \sum_{k=1}^{m-1} n_{k}\left[\int_{0}^{x_{m}} f_{n}^{(k)}\left(\xi_{m}\right) e^{|n|\left(x_{m}-\xi_{m}\right)} d \xi_{m}\right. \\
& \left.-\int_{-\infty}^{x_{m}} f_{n}^{(k)}\left(\xi_{m}\right) e^{|n|\left(\xi_{m}-x_{m}\right)} d \xi_{m}\right] \\
& +C e^{-|n|\left|x_{m}\right|} \quad\left(x_{m}<0\right) .
\end{aligned}
$$

The analogous formula following from (3.27) holds true for $x_{m}>0$.

Denote by $\tilde{f}_{n}^{(k)}(\lambda)$ the Fourier transform of the functions $f_{n}^{(k)}\left(x_{m}\right)(k=$ $1, \ldots, m)$ on $-\infty<x_{m}<+\infty$.

We have

$$
\begin{aligned}
f_{n}^{(k)}\left(x_{m}\right) & =\frac{2}{\sqrt{\pi}} \int_{0}^{+\infty} \tilde{f}_{n}^{(k)}(\lambda) \cos \lambda x_{m} d \lambda, \quad k=1, \ldots, m-1, \\
f_{n}^{(m)}\left(x_{m}\right) & =\frac{2}{\sqrt{\pi}} \int_{0}^{+\infty} \widetilde{f}_{n}^{(m)}(\lambda) \sin \lambda x_{m} d \lambda, \quad k=1, \ldots, m-1 .
\end{aligned}
$$

For example

$$
Z_{m}=-\frac{2}{\sqrt{\pi}} \sum_{n} \cos n_{1} x_{1} \ldots \cos n_{m-1} x_{m-1} \int_{0}^{+\infty} \widetilde{f}_{n}^{(m)}(\lambda) \frac{\sin \lambda x_{m}}{\lambda^{2}+|n|^{2}} d \lambda
$$

belongs to $W_{2}^{(2)}(\Pi)$ and therefore its boundary values are defined for $\partial \Pi$. Moreover they can be estimated by the norm of $f^{(m)}$ in $\mathcal{L}_{2}(\Pi)$. 
Then according to (2.52) (theorem 2.4)

$$
\begin{aligned}
& \int_{B_{R_{0}}}\left|\frac{\partial p}{\partial x_{m}}\right|^{2} r^{\alpha} \zeta d x \\
& \leq\left(1+M_{\gamma}^{2}+\eta\right) m \int_{B_{R_{0}}}|f|^{2} r^{\alpha} \zeta d x \\
& \quad+C\left[\left(\int_{B_{R_{0}}}\left|\frac{\partial p}{\partial x_{m}}\right|^{2} r^{\alpha} \zeta d x\right)^{\frac{m}{m+2 \gamma}}\left(\int_{B_{R_{0}}}|f|^{2} d x\right)^{\frac{2 \gamma}{m+2 \gamma}}+\int_{B_{R_{0}}}|f|^{2} d x\right] .
\end{aligned}
$$

Differentiating $p(x)$ with respect to $x_{k}(k=1, \ldots, m-1)$ we get the same estimates. Then

$$
\begin{aligned}
& \int_{B_{R_{0}}}|\nabla p|^{2} r^{\alpha} \zeta d x \\
& \quad \leq m^{2}\left(1+M_{\gamma}^{2}+\eta\right) \int_{B_{R_{0}}}|f|^{2} r^{\alpha} \zeta d x \\
& \quad+C\left[\left(\int_{B_{R_{0}}}|\nabla p|^{2} r^{\alpha} \zeta d x\right)^{\frac{m}{m+2 \gamma}}\left(\int_{B_{R_{0}}}|f|^{2} d x\right)^{\frac{2 \gamma}{m+2 \gamma}}\right. \\
& \left.\quad+\int_{B_{R_{0}}}|f|^{2} d x\right] .
\end{aligned}
$$

Denote

$$
B_{R_{0}}^{+}=B_{R_{0}} \cap\left(x_{m}>0\right), B_{R_{0}}^{-}=B_{R_{0}} \cap\left(x_{m}<0\right) .
$$

As far as $x_{0} \in B_{R_{0}}^{-}$then for $x \in B_{R_{0}}^{+}$

$$
\left|\bar{x}-x_{0}\right| \leq\left|x-x_{0}\right|
$$

where $\bar{x}$ is symmetric to $x$ with respect to $x_{m}=0$. For $\alpha=2-m-2 \gamma<0$ we have

$$
\left|\bar{x}-x_{0}\right|^{\alpha} \geq\left|x-x_{0}\right|^{\alpha}
$$

and from the monotonicity of $\zeta$ follows

$$
\zeta\left(\left|\bar{x}-x_{0}\right|\right) \geq \zeta\left(\left|x-x_{0}\right|\right) .
$$


Since $f$ and $p$ are expanded on $B_{R_{0}}$ in symmetric and antisymmetric ways we have

$$
\begin{aligned}
\int_{B_{R_{0}}}|f|^{2} r^{\alpha} \zeta d x & =\int_{B_{R_{0}}^{+}}|f|^{2} r^{\alpha} \zeta d x+\int_{B_{R_{0}}^{-}}|f|^{2} r^{\alpha} \zeta d x \\
& \leq 2 \int_{B_{R_{0}}^{-}}|f|^{2} r^{\alpha} \zeta d x .
\end{aligned}
$$

The same estimate is true for the integral $\int_{B_{R_{0}}}|\nabla p|^{2} r^{\alpha} \zeta d x$. Taking into account that

$$
\int_{B_{R_{0}}}|\nabla p|^{2} r^{\alpha} \zeta d x \geq \int_{B_{R_{0}}^{-}}|\nabla p|^{2} r^{\alpha} \zeta d x
$$

we come with the help of (3.29) to

$$
\begin{aligned}
& \int_{B_{R_{0}}^{-}}|\nabla p|^{2} r^{\alpha} \zeta d x \\
& \leq 2 m^{2}\left(1+M_{\gamma}^{2}+\eta\right) \int_{B_{R_{0}}^{-}}|f|^{2} r^{\alpha} \zeta d x \\
& \quad+C\left[\left(\int_{B_{R_{0}}^{-}}|\nabla p|^{2} r^{\alpha} \zeta d x\right)^{\frac{m}{m+2 \gamma}}\left(\int_{B_{R_{0}}^{-}}|f|^{2} d x\right)^{\frac{2 \gamma}{m+2 \gamma}}\right. \\
& \left.\quad+\int_{B_{R_{0}}^{-}}|f|^{2} d x\right] .
\end{aligned}
$$

THEOREM 3.5. - If the conditions of theorem 3.1 are satisfied then the solution of the boundary value problem (3.1), (3.2) satisfies the inequalities

$$
\begin{aligned}
& \int_{\Omega_{R}}|\nabla p|^{2} r^{\alpha} \zeta d x \\
& \leq\left(N_{\gamma}^{2}+\eta\right) \int_{\Omega_{R}}|f|^{2} r^{\alpha} \zeta d x \\
& \quad+C\left[\left(\int_{\Omega_{R}}|\nabla p|^{2} r^{\alpha} \zeta d x\right)^{\frac{m}{m+2 \gamma}}\left(\int_{\Omega_{R}}|f|^{2} d x\right)^{\frac{2 \gamma}{m+2 \gamma}}\right. \\
& \left.\quad+\int_{\Omega_{R}}|f|^{2} d x\right]
\end{aligned}
$$

Vol. 12, $\pi^{\circ}$ 4-1995. 
and

$$
\text { (3.33) } \begin{aligned}
\int_{\Omega_{R}} & \left|D^{2} u\right|^{2} r^{\alpha} \zeta d x \leq \\
\leq & \frac{A_{\alpha, m}^{2}}{\nu^{2}}\left(1+M_{\gamma}^{2}+\eta\right)\left(1+N_{\gamma}\right)^{2} \int_{\Omega_{R}}|f|^{2} r^{\alpha} \zeta d x \\
& +C\left[\left(\int_{\Omega_{R}}\left|D^{2} u\right|^{2} r^{\alpha} \zeta d x\right)^{\frac{m}{m+2 \gamma}}\left(\int_{\Omega_{R}}|D u|^{2} d x\right)^{\frac{2 \gamma}{m+2 \gamma}}\right. \\
& +\int_{\Omega_{R}}|D u|^{2} d x \\
& \left.+\left(\int_{\Omega_{R}}|\nabla p|^{2} r^{\alpha} \zeta d x\right)^{\frac{m}{m+2 \gamma}}\left(\int_{\Omega_{R}}|f|^{2} d x\right)^{\frac{2 \gamma}{m+2 \gamma}}+\int_{\Omega_{R}}|f|^{2} d x\right]
\end{aligned}
$$

where

$$
N_{\gamma}^{2}=\max \left\{\left[1-\frac{\alpha(m-2)}{m-1}\right]\left[1-\frac{\alpha(\alpha+m-2)}{2(m-1)}\right]^{-2}, 2 m^{2}\left(1+M_{\gamma}^{2}\right)\right\}
$$

$\Omega_{R}=\Omega \cap B_{R}\left(x_{0}\right), R$ sufficiently small, $r=\left|x-x_{0}\right|, x_{0} \in \bar{\Omega}, C$ doesn't depend on $x_{0}$ and $\eta$ is an arbitrary small positive number.

Proof. - The inequality (3.32) follows by comparing the estimates (3.4) and (3.31). In fact it is enough to compare the coefficients in front of $\int|f|^{2} r^{\alpha} \zeta d x$ and to take the greatest one. To get (3.33), the system (3.1) should be written in the form

$$
\Delta u=f-\nabla p
$$

and the boundary conditions (3.2) are to be used.

In the interior the inequality follows from the estimate (3.4). In the boundary strip the solution should be continued in an antisymmetric way and estimated with the help of (3.30). The proof of the theorem can now be completed by some simple calculations.

For $\gamma \approx 0$ we have

THEOREM 3.6. - If the conditions of theorem 3.1 are satisfied and the positive $\gamma$ is small, then for the solution of problem (3.1), (3.2) the following estimates 
(3.34) $\quad \int_{\Omega_{H}}|\nabla p|^{2} r^{\alpha} \zeta d x$

$$
\begin{aligned}
\leq & 2 m^{2}\left[1+\frac{m-2}{m+1}+0(\gamma)\right] \int_{\Omega_{R}}|f|^{2} r^{\alpha} \zeta d x \\
& +C\left[\left(\int_{\Omega_{R}}|\nabla p|^{2} r^{\alpha} \zeta d x\right)^{\frac{m}{m+2 \gamma}}\left(\int_{\Omega_{R}}|f|^{2} d x\right)^{\frac{2 \gamma}{m+2 \gamma}}\right. \\
& \left.+\int_{\Omega_{R}}|f|^{2} d x\right],
\end{aligned}
$$

$$
\begin{aligned}
& \int_{\Omega_{R}}\left|D^{2} u\right|^{2} r^{\alpha} \zeta d x \\
& \leq 2\left[1+\frac{m-2}{m+1}+0(\gamma)\right] \\
& \quad \times\left[1+\sqrt{2} m\left(1+\frac{m-2}{m+1}\right)\right]^{2} \int_{\Omega_{R}}|f|^{2} r^{\alpha} \zeta d x \\
& \quad+C\left[\left(\int_{\Omega_{R}}\left|D^{2} u\right|^{2} r^{\alpha} \zeta d x\right)^{\frac{m}{m+2 \gamma}}\left(\int_{\Omega_{R}}|D u|^{2} d x\right)^{\frac{2 \gamma}{m+2 \gamma}}\right. \\
& \quad+\int_{\Omega_{R}}\left(|D u|^{2}+|u|^{2}\right) d x \\
& \quad+\left(\int_{\Omega_{R}}|\nabla p|^{2} r^{\alpha} \zeta d x\right)^{\frac{m}{m+2 \gamma}}\left(\int_{\Omega_{R}}|f|^{2} d x\right)^{\frac{2 \gamma}{m+2 \gamma}} \\
& \left.\quad+\int_{\Omega_{R}}|f|^{2} d x\right]
\end{aligned}
$$

hold.

Consider now the nonstationary system (3.8) with condition (3.9).

THEOREM 3.7. - If $f \in L_{2}\left\{(0, T) ; L_{2, \alpha}(\Omega)\right\}$ with $\alpha$ satisfying the conditions of theorem 3.1 then the solution of system (3.8) with the boundary condition (3.9) satisfies the estimates

(3.36) $\int_{Q_{R}}|\nabla p|^{2} r^{\alpha} \zeta d x d t$

$$
\leq 2 m^{2}\left(N_{\gamma}^{2}+\eta\right) \int_{Q_{R}}|f|^{2} r^{\alpha} \zeta d x d t
$$




$$
\begin{aligned}
& +C\left[\left(\int_{Q_{R}}|\nabla p|^{2} r^{\alpha} \zeta d x d t\right)^{\frac{m}{m+2 \gamma}}\left(\int_{Q_{R}}|f|^{2} d x d t\right)^{\frac{2 \gamma}{m+2 \gamma}}\right. \\
& \left.+\int_{Q_{R}}|f|^{2} d x d t\right]
\end{aligned}
$$

and

$$
\begin{aligned}
\int_{Q_{R}} & \left|D^{2} u\right|^{2} r^{\alpha} \zeta d x d t \\
\leq & \frac{2 A_{\alpha, m}^{2}}{\nu^{2}}\left(1+M_{\gamma}^{2}+\eta\right)\left(1+N_{\gamma}^{2}\right) \int_{Q_{R}}|f|^{2} r^{\alpha} \zeta d x d t \\
& +C\left[\left(\int_{Q_{R}}\left|D^{2} u\right|^{2} r^{\alpha} \zeta d x d t\right)^{\frac{m}{m+2 \gamma}}\left(\int_{Q_{R}}|D u|^{2} d x d t\right)^{\frac{2 \gamma}{m+2 \gamma}}\right. \\
& +\left(\int_{Q_{R}}|\nabla p|^{2} r^{\alpha} \zeta d x d t\right)^{\frac{m}{m+2 \gamma}}\left(\int_{Q_{R}}|f|^{2} r^{\alpha} \zeta d x d t\right)^{\frac{2 \gamma}{m+2 \gamma}} \\
& \left.+\int_{Q_{R}}|f|^{2} d x d t+\int_{Q_{R}}|D u|^{2} d x d t\right]
\end{aligned}
$$

Here $r=\left|x-x_{0}\right|$ and $Q_{R}=(0, T) \times B_{R}\left(x_{0}\right) \cap \Omega$ with sufficiently small $R$. The constant $C$ doesn't depend on $x_{0}$ and $\nu$.

The proof is completely analogous to that of the theorem 3.5 . The only difference is that one has to refer to estimate (2.53).

For small $\gamma>0$ the last two theorems can be formulated in a more explicit way.

THEOREM 3.8. - If the conditions of theorem 3.5 are satisfied, then for the solution of problem (3.1), (3.2) the following estimates

$$
\begin{aligned}
& \int_{\Omega_{R}}|\nabla p|^{2} r^{\alpha} \zeta d x \\
& \leq 2 m^{2}\left[1+\frac{m-2}{m+1}+0(\gamma)\right] \int_{\Omega_{R}}|f|^{2} r^{\alpha} \zeta d x \\
& \quad+C\left[\left(\int_{\Omega_{R}}|\nabla p|^{2} r^{\alpha} \zeta d x\right)^{\frac{m}{m+2 \gamma}}\left(\int_{\Omega_{R}}|f|^{2} d x\right)^{\frac{2 \gamma}{m+2 \gamma}}+\int_{\Omega_{R}}|f|^{2} d x\right]
\end{aligned}
$$




$$
\begin{aligned}
\int_{\Omega_{R}} & \left|D^{2} u\right|^{2} r^{\alpha} \zeta d x \\
\leq & 2\left[1+\frac{m-2}{m+1}+0(\gamma)\right]\left[1+\sqrt{2} m\left(1+\frac{m-2}{m+1}\right)^{1 / 2}\right]^{2} \int_{\Omega_{R}}|f|^{2} r^{\alpha} \zeta d x \\
& +C\left[\left(\int_{\Omega_{R}}\left|D^{2} u\right|^{2} r^{\alpha} \zeta d x\right)^{\frac{2 \gamma}{m+2 \gamma}}\left(\int_{\Omega_{R}}|u|^{2} d x\right)^{\frac{m}{m+2 \gamma}}\left(|D u|^{2}+|u|^{2}\right) d x\right. \\
& \left.+\int_{\Omega_{R}}\left(\int_{\Omega_{R}}|f|^{2} r^{\alpha} \zeta d x\right)^{\frac{m}{m+2 \gamma}}\left(\int_{\Omega_{R}}|f|^{2} d x\right)^{\frac{2 \gamma}{m+2 \gamma}}+\int_{\Omega_{R}}|f|^{2} d x\right]
\end{aligned}
$$

hold.

THEOREM 3.9. - If the conditions of theorem 3.5 are satisfied, then the solution of the problem (3.8), (3.9) satisfies the following inequalities:

$$
\begin{aligned}
\int_{Q_{R}} & |\nabla p|^{2} r^{\alpha} \zeta d x d t \\
\leq & 2 m^{2}\left[1+\frac{m-2}{m+1}+0(\gamma)\right] \int_{Q_{R}}|f|^{2} r^{\alpha} \zeta d x d t \\
& +C\left[\left(\int_{Q_{R}}|\nabla p|^{2} r^{\alpha} \zeta d x d t\right)^{\frac{m}{m+2 \gamma}}\left(\int_{Q_{R}}|f|^{2} d x\right)^{\frac{2 \gamma}{m+2 \gamma}}+\int_{Q_{R}}|f|^{2} d x d t\right] \\
\int_{Q_{R}} & \left|D^{2} u\right|^{2} r^{\alpha} \zeta d x d t \\
\leq & \frac{m}{\nu^{2}}\left[1+\frac{m-2}{m+1}+0(\gamma)\right]\left[1+\sqrt{2} m\left(1+\frac{m-2}{m+1}\right)^{1 / 2}\right]^{2} \int_{Q_{R}}|f|^{2} r^{\alpha} \zeta d x d t \\
& +C\left[\left(\int_{Q_{R}}\left|D^{2} u\right|^{2} r^{\alpha} \zeta d x d t\right)^{\frac{2 \gamma}{m+2 \gamma}}\left(\int_{Q_{R}}|D u|^{2} d x d t\right)^{\frac{m}{m+2 \gamma}}\right. \\
& +\left(\int_{Q_{R}}|\nabla p|^{2} r^{\alpha} \zeta d x d t\right)^{\frac{2 m}{m+2 \gamma}} \\
& \left.\times\left(\int_{Q_{R}}|f|^{2} d x d t\right)^{\frac{2 \gamma}{m+2 \gamma}}+\int_{Q_{R}}|p|^{2} d x d t\right],(m \geq 3)
\end{aligned}
$$




\section{REGULARITY OF SOLUTIONS FOR DEGENERATED ELLIPTIC SYSTEMS}

In a bounded domain $\Omega \subset R^{m}(m \geq 2)$ we consider a system

$$
L(u) \equiv \sum_{i=0}^{m} D_{i} a_{i}(x, D u)=0,
$$

where $u$ and $a_{i}(x, p)(i=0,1, \ldots, m)$ are $\mathrm{N}$-dimensional vector functions with components $u^{(k)}(x), a_{i}^{(k)}(x, p)(k=1, \ldots, N)$,

$D u=\left(D_{0} u, D_{1} u, \ldots, D_{m} u\right), \quad D_{i}=\frac{\partial}{\partial x_{i}}(i=1, \ldots, m)$ and $D_{0}=-I$,

(now, different from $\S$, we include $u$ in $D u$ ). About the functions $a_{i}(x, p)$ we assume, that they satisfy the following conditions:

(1) All $a_{i}(x, p)$ satisfy the Caratheodory conditions and are differentiable with respect to variables $p$;

(2) The $(m+1) N \times(m+1) N$ matrix

$$
A=\left\{\frac{\partial a_{i}^{(k)}}{\partial p_{j}^{(l)}}\right\} \quad(i, j=0,1, \ldots, m ; k, l=1, \ldots, N ;)
$$

is symmetric and the eigenvalues of this matrix satisfy the inequalities

$$
\frac{\lambda}{1+|p|^{s}} \leq \lambda_{j}(x, p) \leq \frac{\Lambda}{1+|p|^{s}}
$$

where $\lambda, \Lambda=$ const $>0$ and $0 \leq s<1$;

(3) For arbitrary $u \in W_{q}^{(1)}(\Omega)(q>1)$ the result of the substitution $a_{i}(x, D u(x))(i=0, \ldots, m)$ will belong to $L_{q /(1-s)}(\Omega)$;

(4) The inequality

$$
\left|\frac{\partial a_{i}}{\partial x_{k}}\right| \leq C|p|+b \quad(i=1, \ldots, m)
$$

holds, where $b$ is a sufficiently small nonnegative value;

(5) For all $u \in W_{q}^{(2)}(\Omega)$ the result of substitution in $L(u)$ belongs to $L_{q}(\Omega)$.

Consider the solution of (4.1) with the boundary condition

$$
\left.u\right|_{\partial \Omega}=0 \text {. }
$$


In [3] (chapter $1, \S 4)$ it was proved that the universal iterational process

$$
\begin{gathered}
\Delta u_{n+1}-u_{n+1}=\Delta u_{n}-u_{n}-\Lambda^{-1} L\left(u_{n}\right), \\
\left.u_{n}\right|_{\partial \Omega}=0(n=0,1, \ldots,)
\end{gathered}
$$

converges in $W_{2-s}^{(1)}(\Omega)$ to the weak solution $u$ of (4.1), (4.5) if $u \in W_{2}^{(1)}(\Omega)$.

Consider also the process (4.6) with a penalty term,

$$
\Delta u_{n+1}-u_{n+1}=\Delta u_{n}-u_{n}-\Lambda^{-1}\left[\delta \Delta u_{n}+L\left(u_{n}\right)\right] \quad(\delta \geq 0),
$$

with the same condition (4.5).

In [3] it was also shown that a subsequence of the iterations of process (4.7) converges weakly to the solution. So, if we want to show that the solution has Hölder continuous first derivatives it is enough to show that the iterations of $(4.6)$ or (4.7) satisfy the inequality

$$
\int_{\Omega_{R}}\left|D^{2} u_{n}\right|^{2} r^{\alpha} d x \leq C,
$$

where $\Omega_{R}=B_{R}\left(x_{0}\right) \cap \Omega, x_{0} \in \bar{\Omega}, \alpha=2-m-2 \gamma(0<\gamma<1), r=\left|x-x_{0}\right|$, and $C$ doesn't depend on $x_{0}$ and $n$. It is also assumed that $R$ is sufficiently small and fixed.

LEMMA 4.1. - If the conditions 1)-3) are satisfied and $u_{0}(x) \in \stackrel{\circ}{W}_{2}^{(1)}(\Omega)$ then

$$
\begin{aligned}
& \left(\int_{\Omega}\left|D u_{n+1}\right|^{2} d x\right)^{1 / 2} \\
& \quad \leq\left(1-\frac{\lambda \Lambda^{-1}}{1+\left[\max \left\{\sup _{\Omega}\left|D u_{n}\right|, \sup _{\Omega}\left|D u_{n+1}\right|\right\}\right]^{s}}\right)^{1 / 2} \\
& \quad \times\left(\int_{\Omega}\left|D u_{n}\right|^{2} d x\right)^{1 / 2}+\Lambda^{-1}|a|
\end{aligned}
$$

holds, where

$$
|a|^{2}=\int_{\Omega} \sum_{i=0}^{m}\left|a_{i}(x, 0)\right|^{2} d x .
$$

Proof. - Multiply both sides of the system (4.6) by $u_{n+1}$ and integrate once by parts. Then

$$
\int_{\Omega} D u_{n+1} D u_{n+1} d x=\int_{\Omega}\left[D_{i} u_{n}-\Lambda^{-1} a_{i}\left(x, D u_{n}\right)\right] D_{i} u_{n+1} d x
$$


where summation as always runs over repeated indices.

Adding and subtracting $a_{i}(x, 0)$ under the square brackets on the right hand side, we get

$$
\begin{aligned}
\int_{\Omega} D & u_{n+1} D u_{n+1} d x \\
= & \int_{\Omega}\left[D_{i} u_{n}-\Lambda^{-1}\left(a_{i}\left(x, D u_{n}\right)-a_{i}(x, 0)\right)\right] D_{i} u_{n+1} d x \\
& -\int_{\Omega} a_{i}(x, 0) D_{i} u_{n+1} d x .
\end{aligned}
$$

Applying the mean value theorem we come to

$$
\begin{aligned}
\int_{\Omega}\left|D u_{n+1} D u_{n+1}\right| d x= & \int_{\Omega}\left(I-\Lambda^{-1} \bar{A}\right) D u_{n} \cdot D u_{n+1} d x \\
& -\int_{\Omega} a_{i}(x, 0) D_{i} u_{n+1} d x
\end{aligned}
$$

where $\bar{A}$ denotes the matrix $A$ with intermediate values of variables.

The Hölder inequality gives

$$
\left(\int_{\Omega}\left|D u_{n+1}\right|^{2} d x\right)^{1 / 2} \leq \sup _{\Omega} \|\left(I-\Lambda^{-1} \bar{A} \|\left(\int_{\Omega}\left|D u_{n}\right|^{2} d x\right)^{1 / 2}+|a|\right.
$$

It can be easily proved (see for example [3] p. 58, (2.29)), that

$$
\sup _{\Omega}\left\|I-\Lambda^{-1} \bar{A}\right\| \leq \sup _{i, \Omega}\left|1-\Lambda^{-1} \bar{\lambda}_{i}\right|
$$

Using the right side of the inequalities (4.3) we get

$$
\left\|I-\Lambda^{-1} \bar{A}\right\| \leq 1-\frac{\lambda \Lambda^{-1}}{1+\left[\max \left\{\sup _{\Omega}\left|D u_{n}\right|, \sup _{\Omega}\left|D u_{n+1}\right|\right\}\right]^{s}} .
$$

Suppose that the cut-off function $\zeta(r)(2.46)$ satisfies in addition the inequality

$$
\left|\zeta^{\prime}\right||\zeta|^{-1 / 2}<C
$$

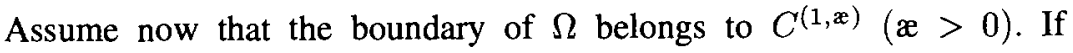
conditions 4) and 5) are satisfied and $u_{0}$ (the initial iteration of (4.6) or (4.7)) belongs to $W_{q}^{(2)}(\Omega) \cap \dot{W}_{q}^{(1)}(\Omega)(q>1)$, then all iterations belong 
to the same space. The iterations can be extented outside the domain $\Omega$ to a sufficiently narrow strip preserving the class. This can be made with the help of the well-known procedure which we have used in the previous paragraph. First one considers a plane piece of the boundary and expands all of the $u_{n}$ in an antisymmetric way. This gives one the same class of $W_{q}^{(2)}\left(\Omega \cup \Omega_{R}\right)$ for balls $B_{R}\left(x_{0}\right)$ which don't completely lie in $\Omega$. As we have shown in [3] (chapter $4, \S 3$ ) all the conditions 1) - 5) don't change, and the values, $s, \lambda$ and $\Lambda$ will be the same. This gives also us the possibility to consider only the case when $\Omega_{R}=\Omega \cap B_{R}\left(x_{0}\right)=B_{R}\left(x_{0}\right)$ and this gives the fixed small $R_{0}$.

LEMMA 4.2. - If the conditions 1)-5) are satisfied and $u_{0} \in W_{q}^{(2)}(\Omega) \cap$ $\dot{W}_{q}^{(1)}(\Omega)(q>2)$ then the iterations (4.6) or (4.7) satisfy the inequality

$$
\begin{aligned}
\int_{\Omega_{R}} & \left|D^{2} u_{n+1}\right|^{2} \zeta d x d t \\
\leq & {\left[1-\frac{\lambda \Lambda^{-1}}{1+\left[\max \left\{\sup _{\Omega}\left|D u_{n}\right|, \sup _{\Omega}\left|D u_{n+1}\right|\right\}\right]^{s}}+\eta\right] } \\
& \times\left.\int_{\Omega_{R}} D^{2} u_{n}\right|^{2} \zeta d x d t+C|a|^{2},
\end{aligned}
$$

where $C$ doesn't depend on $x_{0} \in \bar{\Omega}, n$ and in the case of (4.7) on $\delta$.

Proof. - According to our previous consideration we can suppose that $\Omega_{R}=B_{R}\left(x_{0}\right)$. Multiply (4.6) (or 4.7) by $\Delta u_{n+1} \zeta$ and integrate by parts as in the proof of lemma 1.2 or lemma 1.5 for $\alpha=0$. In [3] (theorem 1) it is shown that if $\zeta$ satisfies (4.13) then

$$
\begin{aligned}
\int_{B_{R}}\left|D u_{n+1}\right|^{2} \zeta d x \leq & \sum_{k=1}^{m} \int_{B_{R}}\left(I-\Lambda^{-1} \bar{A}\right) D D_{k} u_{n} D D_{k} u_{n+1} \zeta d x \\
& +C|a|\left(\int_{B_{R}}\left|D u_{n+1}\right|^{2} \zeta d x\right)^{1 / 2}
\end{aligned}
$$

From this and from (4.12) immediately follows (4.14).

Let $w_{k}(x)$ satisfy the equation

$$
\Delta w_{k}=\Delta u_{n+1} \cdot r^{\alpha_{k}} \zeta
$$

and the boundary condition

$$
\left.w_{k}\right|_{\partial B_{R}}=0(k=1,2, \ldots, M)
$$

Vol. $12, \mathrm{n}^{\circ} 4-1995$. 
where $M$ is a positive integer and $\alpha_{k}$ is monotone and satisfy the following relations:

$$
\left\{\begin{array}{c}
\alpha_{1}=-m / 2+\eta, \\
0<\alpha_{k-1}-2 \alpha_{k}<m, \\
\alpha_{k} \notin[2-m, 3-m], \alpha_{M-1}>2-m \\
\alpha_{M}=\alpha=2-m-2 \gamma(0<\gamma<1) .
\end{array}\right.
$$

According to results of E. M. Stein [5] and V. A. Kondratjev [8] the inequality

$$
\int_{B_{R}}\left(\left|D^{2} w\right|^{2}+|D w|^{2}+|w|^{2}\right) r^{\beta} d x \leq C \int_{B_{R}}|\Delta w|^{2} r^{\beta} d x
$$

holds, if $-m<\beta<m$ and $w=0$ on $\partial B_{R}$.

Multiply (4.6) or (4.7) by $\Delta w_{k} \zeta$ and integrate twice by parts. It is obvious that $\zeta^{2}$ also satisfies (2.46) and (4.13). Then we get

$$
\begin{aligned}
\int_{B_{R}} & \left|\Delta u_{n+1}\right|^{2} r^{\alpha_{k}} \zeta^{2} d x \\
= & \int_{B_{R}}\left\{u_{n, i, j}-\Lambda^{-1}\left[a_{i}\left(x, D u_{n}\right)\right]_{j}\right\} w_{k, i, j} \zeta^{2} d x \\
& +\int_{B_{R}}\left[u_{n, i}-\Lambda^{-1} a_{i}\left(x, D u_{n}\right)\right] w_{k, i j}\left(\zeta^{2}\right)_{j} d x-\int_{B_{R}}\left[u_{n, i}\right. \\
& \left.-\Lambda^{-1} a_{i}\left(x, D u_{n}\right)\right] w_{k, i j}\left(\zeta^{2}\right)_{i} d x+\ldots=I_{1}+I_{2}++I_{3}
\end{aligned}
$$

(the unwritten terms contain only the first derivatives of $u_{n}$ and $w_{k}$ ). Let us estimate at first the integral $I_{1}$. It is easy to see that

$$
\begin{aligned}
(4.20) I_{1}= & \int_{B_{R}}\left\{u_{n, i j}-\Lambda^{-1}\left[a_{i}\left(x, D u_{n}\right)\right]_{j}\right\} w_{k, i j} \zeta^{2} d x \\
\leq & \sup _{\bar{\Omega}}\left\|I-\Lambda^{-1} \bar{A}\right\| \\
& \times\left(\int_{B_{R}}\left|D^{2} u_{n}\right|^{2} r^{\alpha_{k}} \zeta^{2} d x\right)^{\frac{1}{2}}\left(\int_{B_{R}}\left|D^{2} w_{k}\right|^{2} r^{-\alpha_{k}} \zeta^{2} d x\right)^{\frac{1}{2}}+\ldots
\end{aligned}
$$


Furthermore,

$$
\begin{aligned}
& \int_{B_{R}}\left|D^{2} w_{k}\right|^{2} r^{-\alpha_{k}} \zeta^{2} d x \\
& =\sum_{i, j=1}^{m} \int_{B_{R}} w_{k, i j}^{2} r^{-\alpha_{k}} \zeta^{2} d x \\
& =\sum_{i, j=1}^{m} \int_{B_{R}}\left[\left(w_{k} \zeta\right)_{i j}-\left(w_{k, j} \zeta_{i}+w_{k, i} \zeta_{j}\right)-w_{k} \zeta_{i j}\right]^{2} r^{-\alpha_{k}} d x \\
& \leq \int_{B_{R}}\left|D^{2}\left(w_{k} \zeta\right)\right|^{2} r^{-\alpha_{k}} d x \cdot(1+\eta) \\
& \quad+C \int_{B_{R}}\left(\left|D w_{k}\right|^{2}+\left|w_{k}\right|^{2}\right) r^{-\alpha_{k}}\left|D^{2} \zeta\right|^{2} d x .
\end{aligned}
$$

According to the inequality of S. Chelkak ([9], p. 28, Lemma 1.2), we have

$$
\int_{B_{R}}\left|D^{2}\left(w_{k} \zeta\right)\right|^{2} r^{\alpha_{k}} d x \leq\left[1-\frac{4 \alpha_{k}(m-1)}{\left(\alpha_{k}+m\right)^{2}}\right] \int_{B_{R}}\left|\Delta\left(w_{k} \zeta\right)\right|^{2} r^{-\alpha_{k}} d x
$$

Then, from (4.16) and the fact that $D \zeta \equiv 0$ for $r \leq R / 2$ it follows that

$$
\begin{aligned}
\int_{B_{R}}\left|D^{2} w_{k}\right|^{2} r^{-\alpha_{k}} \zeta^{2} d x \leq & {\left[1-\frac{4 \alpha_{k}(m-1)}{\left(\alpha_{k}+m\right)^{2}}\right] \int_{B_{R}}\left|\Delta w_{k}\right|^{2} r^{-\alpha_{k}} \zeta^{2} d x } \\
& +C \int_{B_{R}}\left(\left|D w_{k}\right|^{2}+\left|w_{k}\right|^{2}\right) r^{-\alpha_{k}}\left|D^{2} \zeta\right|^{2} d x \\
\leq & {\left[1-\frac{4 \alpha_{k}(m-1)}{\left(\alpha_{k}+m\right)^{2}}\right] \int_{B_{R}}\left|\Delta u_{n+1}\right|^{2} r^{-\alpha_{k}} \zeta^{2} d x } \\
& +C \int_{B_{R}}\left(\left|D w_{k}\right|^{2}+\left|w_{k}\right|^{2}\right) r^{-2 \alpha_{k}+\alpha_{k-1}} d x .
\end{aligned}
$$

If $\alpha_{k}$ satisfies (4.17) we can apply (4.18) and come to the inequality

$$
\begin{aligned}
& \int_{B_{R}}\left|D^{2} w_{k}\right|^{2} r^{-\alpha_{k}} \zeta^{2} d x \\
& \quad \leq\left[1-\frac{4 \alpha_{k}(m-1)}{\left(\alpha_{k}+m\right)^{2}}\right] \int_{B_{R}}\left|\Delta u_{n+1}\right|^{2} r^{\alpha_{k}} \zeta^{2} d x \\
& \quad+C\left(\int_{B_{R}}\left|\Delta u_{n+1}\right|^{2} r^{\alpha_{k-1}} \zeta^{2} d x+\int_{B_{R}}\left|D u_{n}\right|^{2} d x\right) .
\end{aligned}
$$

Vol. $12, \mathrm{n}^{\circ} 4-1995$. 
Carrying out the same considerations for $I_{2}$ and $I_{3},(4.12),(4.19)$ and (4.20) yield the relation

$$
\begin{aligned}
\int_{B_{R}} & \left|\Delta u_{n+1}\right|^{2} r^{\alpha_{k}} \zeta^{2} d x \\
\leq & {\left[1-\frac{4 \alpha_{k}(m-1)}{\left(\alpha_{k}+m\right)^{2}}+\eta\right] } \\
& \times\left(1-\frac{\lambda \Lambda^{-1}}{1+\left[\max \left\{\sup _{\Omega}\left|D u_{n}\right|, \sup _{\Omega}\left|D u_{n+1}\right|\right\}\right]^{s}}\right) \\
& \times \int_{B_{R}}\left|D^{2} u_{n}\right|^{2} r^{\alpha_{k}} \zeta^{2} d x \\
& +C\left(|a|^{2}+\int_{B_{R}}\left|\Delta u_{n+1}\right|^{2} r^{\alpha_{k}-1} \zeta^{2} d x+\int_{B_{R}}\left|D u_{n}\right|^{2} d x\right) .
\end{aligned}
$$

Inequality (2.52) (th. 2.4) gives for $k=M$

$$
\begin{aligned}
& \int_{B_{R}}\left|D^{2} u_{n+1}\right|^{2} r^{\alpha} \zeta^{2} d x \\
& \quad \leq\left(1+M_{\gamma}^{2}\right)\left[1-\frac{4 \alpha(m-1)}{(\alpha+m)^{2}}+\eta\right] \\
& \quad \times\left(1-\frac{\lambda \Lambda^{-1}}{\left.1+\left[\max _{\Omega} \sup _{\Omega}\left|D u_{n}\right|, \sup _{\Omega}\left|D u_{n+1}\right|\right\}\right]^{s}}\right) \\
& \quad \times \int_{B_{R}}\left|D^{2} u_{n}\right|^{2} r^{\alpha} \zeta^{2} d x \\
& \quad+C\left[|a|^{2}+\int_{B_{R}}\left|D u_{n}\right|^{2} d x+\int_{B_{R}}\left|\Delta u_{n+1}\right|^{2} r^{\alpha_{M-1}} \zeta^{2} d x\right. \\
& \left.\quad+\left(\int_{B_{R}}\left|D^{2} u_{n+1}\right|^{2} r^{\alpha} \zeta^{2} d x\right)^{\frac{m}{m+2 \gamma}} \cdot\left(\int_{B_{R}}\left|D u_{n}\right|^{2} d x\right)^{\frac{2 \gamma}{m+2 \gamma}}\right]
\end{aligned}
$$

For $k<M$ according to [2] (p. 51, lemma 2.2) (see also [8])

$$
\begin{aligned}
& \int_{B_{R}}\left|D^{2} u_{n+1}\right|^{2} r^{\alpha_{k}} \zeta^{2} d x \\
& \leq {\left[1-\frac{4 \alpha_{k}(m-1)}{\left(\alpha_{k}+m\right)^{2}}+\eta\right] } \\
& \times\left(1-\frac{\lambda \Lambda^{-1}}{1+\left[\max \left\{\sup _{\Omega}\left|D u_{n}\right|, \sup _{\Omega}\left|D u_{n+1}\right|\right\}\right]^{s}}\right)
\end{aligned}
$$




$$
\begin{aligned}
& \times \int_{B_{R}}\left|D^{2} u_{n}\right|^{2} r^{\alpha_{k}} \zeta^{2} d x \\
& +C\left(\int_{B_{R}}\left|D u_{n}\right|^{2} d x+|a|^{2}+\int_{B_{R}}\left|\Delta u_{n+1}\right|^{2} r^{\alpha_{k-1}} \zeta^{2} d x\right) .
\end{aligned}
$$

THEOREM 4.1. - Suppose the conditions 1)-5) are satisfied and the inequalities

$$
\left\{\begin{array}{c}
\int_{\Omega}\left|D u_{0}\right|^{2} d x<\eta_{0}^{2}, \\
\int_{\Omega}\left|D^{2} u_{0}\right|^{2} r^{\alpha_{k}} d x<\eta_{k}^{2} \quad(k=1, \ldots, M-1), \\
|a|^{2}+\sum_{j=1}^{k-1} \eta_{j}^{2}<\varepsilon \eta_{k}^{2}
\end{array}\right.
$$

hold true $\left(\varepsilon, a, \eta_{k}, b=\right.$ const $>0$ are suficiently small numbers $)$. If the relation

$$
\frac{\Lambda}{\lambda} \frac{\left(1+M_{\gamma}^{2}\right)\left[1-\frac{4 \alpha(m-1)}{(\alpha+m)^{2}}\right]-1}{\left(1+M_{\gamma}^{2}\right)\left[1-\frac{4 \alpha(m-1)}{(\alpha+m)^{2}}\right]}<1
$$

is satisfied, then the solution of the problem (4.1), (4.5) belongs to $C^{1, \gamma}(\bar{\Omega})$ with $\gamma=-(\alpha+m-2) / 2$ and the subsequences of iterations of (4.6) and (4.7) converge to this solution.

Proof. - Consider at first the case $m \geq 4$. As we have mentioned before it is sufficient to prove inequality (4.8). Suppose that $u \in W_{q}^{(2)}(\Omega)$ with $q>m(m+\alpha)^{-1}$.

Then all $u_{n}$ are in $W_{q}^{(2)}(\Omega)$. From this follows that $\forall u_{n} \in W_{2, \alpha}^{(2)}(\Omega)$.

If we write (2.49) for the functions $u_{j} \zeta$, we get

$$
\begin{aligned}
& \left|u_{j, i}\left(x_{0}\right)\right|^{2} \\
& \leq C\left(|a|^{2}+\sum_{j=0}^{M-1} \eta_{j}^{2}\right)^{\frac{2 \gamma}{m+2 \gamma}}\left[\left(\int_{B_{R}}\left|D^{2} u_{0}\right|^{2} r^{\alpha} \zeta^{2} d x\right)^{\frac{m}{m+2 \gamma}}\right. \\
& \left.\quad+\left(|a|^{2}+\sum_{j=0}^{M-1} \eta_{j}^{2}\right)^{\frac{m}{m+2 \gamma}}\right] \quad(j=0,1 ; i=1, \ldots, m),
\end{aligned}
$$


where $u_{j, i}=D_{i} u_{j}$ In fact, from (2.49) and $u=u_{j} \zeta$ we obtain after some calculations that

$$
\begin{aligned}
\left|u_{j, i}\left(x_{0}\right)\right|^{2} \leq & C\left(\int_{\Omega}\left|D u_{j}\right|^{2} d x\right)^{\frac{2 \gamma}{m+2 \gamma}} \\
\times & {\left[\left(\int_{B_{R}}\left|D^{2} u_{j}\right|^{2} r^{\alpha} \zeta^{2} d x\right)^{\frac{m}{m+2 \gamma}}+\left(\int_{\Omega}\left|D u_{j}\right|^{2} d x\right)^{\frac{m}{m+2 \gamma}}\right] } \\
(j=0,1) . &
\end{aligned}
$$

Now (4.25) follows from (4.23) for $j=0$. Applying (4.9) and the inequality $(a+b)^{2} \leq 2\left(a^{2}+b^{2}\right)$, we have

$$
\int_{\Omega}\left|D u_{1}\right|^{2} d x \leq 2\left(\int_{\Omega}\left|D u_{0}\right|^{2} d x+|a|^{2}\right) \leq 2\left(\eta_{0}^{2}+|a|^{2} \Lambda^{-2}\right) .
$$

After using (4.21) and (4.22), the inequalitiy $|a b|<\eta a^{2}+4^{-1} \eta^{-1} b^{2}$ the estimates give the relation

$$
\int_{B_{R}\left(x_{0}\right)}\left|D^{2} u_{1}\right|^{2} r^{\alpha} \zeta^{2} d x \leq C\left[\int_{B_{R}\left(x_{0}\right)}\left|D^{2} u_{0}\right|^{2} r^{\alpha} \zeta^{2} d x+|a|^{2}+\sum_{j=0}^{M-1} \eta_{j}^{2}\right]
$$

Therefore

$$
\begin{aligned}
\left|u_{1, i}\left(x_{0}\right)\right|^{2} \leq & C\left(\eta_{0}^{2}+|a|^{2}\right)^{\frac{2 \gamma}{m+2 \gamma}}\left[\left(\int_{B_{R}\left(x_{0}\right)}\left|D^{2} u_{0}\right|^{2} r^{\alpha} \zeta d x\right)^{\frac{m}{m+2 \gamma}}\right. \\
& \left.+\left(|a|^{2}+\sum_{k=1}^{M-1} \eta_{k}^{2}\right)^{\frac{m}{m+2 \gamma}}\right]
\end{aligned}
$$

From (4.25) follows that for $j=0,1$

$$
\begin{aligned}
\sup _{\Omega} & \left|D u_{j}\right|^{2} \\
\leq & C\left(|a|^{2}+\sum_{k=0}^{M-1} \eta_{k}^{2}\right)^{\frac{2 \gamma}{m+2 \gamma}} \\
& \times\left[\left(\sup _{x_{0} \in \bar{\Omega}} \int_{B_{R}\left(x_{0}\right)}\left|D^{2} u_{0}\right|^{2} r^{\alpha} \zeta d x\right)^{\frac{m}{m+2 \gamma}}+\left(|a|^{2}+\sum_{k=0}^{M-1} \eta_{k}^{2}\right)^{\frac{m}{m+2 \gamma}}\right] .
\end{aligned}
$$


Take $|a|^{2}+\sum_{j=0}^{M-1} \eta_{j}^{2}$ so small that $C\left(|a|^{2}+\sum_{j=0}^{M-1} \eta_{j}^{2}\right)^{\frac{2 \gamma}{m+2 \gamma}}<1$. Then (4.21) gives

$$
\begin{aligned}
& \sup _{x_{0} \in \Omega} \int_{B_{R}}\left|D^{2} u_{1}\right|^{2} r^{\alpha} \zeta^{2} d x \\
& \leq\left(1+M_{\gamma}^{2}\right)\left[1-\frac{4 \alpha(m-1)}{(\alpha+m)^{2}}+\eta\right] \\
& \quad \times\left\{1-\frac{\lambda \Lambda^{-1}}{1+\left[\left(\sup _{x_{0} \in \bar{\Omega}} \int_{B_{R}\left(x_{0}\right)}\left|D^{2} u_{0}\right|^{2} r^{\alpha} \zeta^{2} d x\right)^{\frac{m}{m+2 \gamma}}+C\left(|a|^{2}+\sum_{k=0}^{M-1} \eta_{k}^{2}\right)^{\frac{m}{2(m+2 \gamma)}}\right]^{s}}\right\} \\
& \times \sup _{x_{0} \in \bar{\Omega}} \int_{B_{R}}\left|D^{2} u_{0}\right|^{2} r^{\alpha} \zeta^{2} d x+C\left(|a|^{2}+\sum_{j=1}^{M-1} \eta_{j}^{2}\right) .
\end{aligned}
$$

Set

$$
\left\{\begin{array}{c}
X_{l}=\sup _{x_{0} \in \Omega} \int_{B_{R}}\left|D^{2} u_{l}\right|^{2} r^{\alpha} \zeta^{2} d x,(l=0,1) \\
Q=\left(1+M_{\gamma}^{2}\right)\left[1-\frac{4 \alpha(m-1)}{(\alpha+m)^{2}}+\eta\right] \\
H=C\left(|a|^{2}+\sum_{k=0}^{M-1} \eta_{k}^{2}\right) .
\end{array}\right.
$$

Inequality (4.26) now turns to

$$
X_{1} \leq Q\left(1-\frac{\lambda \Lambda^{-1}}{1+X_{0}^{\frac{m s}{2(m+2 \gamma)}}+H^{\frac{m s}{2(m+2 \gamma)}}}\right) X_{0}+H
$$

which can be written in the form

$$
X_{1} \leq X_{0}+(Q-1)\left\{\left[1-\frac{Q \lambda \Lambda^{-1}}{(Q-1)\left(1+X_{0}^{\frac{m s}{2(m+2 \gamma)}}+H^{\frac{m s}{2(m+2 \gamma)}}\right)}\right] X_{0}+\frac{H}{Q-1}\right\} .
$$

Let the condition

$$
Q \lambda \Lambda^{-1}(Q-1)^{-1}>1,
$$

holds. Then there exists such a $q_{0} \in(0,1)$ that $Q \lambda \Lambda^{-1}\left(Q-q_{0}\right)>1$. Let $H$ be so small that $H \leq\left(1-q_{0}\right)\left[Q \lambda \Lambda^{-1}\left(Q-q_{0}\right)-1-H^{\frac{m s}{2(m+2 \gamma)}}\right]^{\frac{2(m+2 \gamma)}{m s}} \equiv$ Vol. $12, \mathrm{n}^{\circ} 4-1995$. 
$P\left(1-q_{0}\right)$. Then after small calculations we get from the inequality $X_{0} \leq P$ the relation

$$
X_{1} \leq P
$$

Let us return now to (4.22). From (4.25) and $C\left(|a|^{2}+\sum_{k=1}^{M-1} \eta_{k}^{2}\right)^{\frac{2 \gamma}{m+2 \gamma}}<1$ we get

$$
\begin{aligned}
& \int\left|D^{2} u_{1}\right|^{2} r^{\alpha_{k}} \zeta^{2} d x \\
& \leq\left[1-\frac{4 \alpha_{k}(m-1)}{\left(\alpha_{k}+m\right)^{2}}+\eta\right] \\
& \quad \times\left(1-\frac{\lambda \Lambda^{-1}}{1+\left[\left(\sup _{x_{0} \in \Omega} \int_{B_{R}}\left|D^{2} u_{0}\right|^{2} r^{\alpha} \zeta^{2} d x\right)^{\frac{m}{m+2 \gamma}}+C\left(|a|^{2}+\sum_{j=0}^{M-1} \eta_{j}^{2}\right)^{\frac{m}{2(m+2 \gamma)}}\right]^{s}}\right) \\
& \times \int_{B_{R}}\left|D^{2} u_{0}\right|^{2} r^{\alpha_{k}} \zeta^{2} d x \\
& +C\left[\int_{B_{R}}\left|D u_{0}\right|^{2} d x+|a|^{2}+\int|\Delta u|^{2} r^{\alpha_{k-1}} \zeta^{2} d x\right] .
\end{aligned}
$$

With the help of (4.27) and (4.29) we have for $k<M$

$$
\begin{aligned}
\int_{B_{R}\left(x_{0}\right)}\left|D^{2} u_{1}\right|^{2} r^{\alpha_{k}} \zeta d x & \\
\leq & {\left[1-\frac{4 \alpha_{k}(m-1)}{\left(\alpha_{k}+m\right)^{2}}+\eta\right]\left[1-\frac{4 \alpha(m-1)}{(\alpha+m)^{2}}\right]^{-1} } \\
& \times\left(1+M_{\gamma}^{2}\right)^{-1} \int_{B_{R}\left(x_{0}\right)}\left|D^{2} u_{0}\right|^{2} r^{\alpha_{k}} \zeta d x+C\left(|a|^{2}+\sum_{j=0}^{k-1} \eta_{j}^{2}\right) .
\end{aligned}
$$

All $\alpha_{k}$ are negative and decreasing. Then from the last inequality we obtain

$$
\int_{B_{R}\left(x_{0}\right)}\left|D^{2} u_{1}\right|^{2} r^{\alpha_{k}} \zeta d x \leq\left(1+M_{\gamma}^{2}\right)^{-1} \eta_{k}^{2}+C\left(|a|^{2}+\sum_{j=0}^{k-1} \eta_{j}^{2}\right) .
$$

From (4.23) follows that

$$
\int_{B_{R}\left(x_{0}\right)}\left|D^{2} u_{1}\right|^{2} r^{\alpha_{k}} \zeta d x<\eta_{k}^{2}
$$


and therefore for $u_{1}$ all conditions of the theorem are satisfied. Thus inequality (4.8) and the theorem are proved for $m \leq 4$.

For $m=2$ and $m=3$ let us remark that if we take $\alpha_{1}=-\frac{m}{2}+\eta$ then the condition

$$
-\frac{m}{2}+\eta<2-m-2 \gamma
$$

can be satisfied at least for small $\gamma$ and all consideration are simplified.

Remark 4.1. - If $\gamma>0$ is small then the condition (4.24) gives

$$
\frac{\Lambda}{\lambda} \frac{\left(1+\frac{m-2}{m+1}\right)[1+(m-2)(m-1)]-1}{\left(1+\frac{m-2}{m+1}\right)[1+(m-2)(m-1)]}<1
$$

For $m=2$ this inequality does not restrict the dispersion of the spectrum for the matrix of ellipticity.

\section{REFERENCES}

[1] H. O. CORDES, Uber die erste Randwertaufgabe bei quasilinearen Differentialgleichungen zweiter Ordnung in mehr als zwei Variablen, Math. Ann., Bd. 131, 1956, pp. 278-312.

[2] A. Koshelev, About some coercive inequalities for elementary elliptic and parabolic operators. Institut für Angewandte Analysis und Stochastik, preprint No. 15, Berlin 1992.

[3] A. Koshelev, Reguljarnost reschenyi ellipticheskih uravnenij i sistem, Moskva, Nauka, 1986.

[4] A. Koshelev, Regularity of solutions for some quasilinear parabolic systems, Mathematische Nachrichten, Vol. 162, 1993, pp. 59-88.

[5] E. M. 'Sten, Singular integrals and differentiability properties on functions, Princeton University Press, New Jersey, 1970.

[6] V. A. SolonNIKov, On general boundary value problems for elliptic systems in the sense of Douglis-Nirenberg,' I, Izv, Akad. Nauk SSSR, ser. mat., Vol. 28, 1964, pp. 665-706; II, Trudy Mat. Inst. steklov, Vol. 70, 1966, pp. 133-212.

[7] A. KOSHELEV, A priori estimates in $L_{p}$ and the existence theorems, Uspehi matem. nauk, Vol. 13:4, 1958, pp. 29-88.

[8] V. A. KondRAT'EV, Solvability of the first boundary-value problem for strongly elliptic equations, Trans. Moscow Math. Soc., Vol. 16, 1967, pp. 209-292.

[9] S. I. Chelkak and A. I. Kosheley, Regularity of solutions of quasilinear elliptic systems, Leipzig: Teubner, 1985.

[10] O. A. LADYZHENSKAYA, The mathematical theory of viscous incompressible flow, Gordon and Breach, New York, London, Paris, 1969.

(Manuscript received August, 1993;

Revised version received November 25, 1993.) 